\title{
Pierre, fils de Jean II, duc de Bretagne ou l'existence mouvementée d'un noble cadet dissipateur
}

Peter, son of John II, duke of Brittany, or the tumultuous life of a younger son

Marjolaine Lémeillat

\section{OpenEdition}

Journals

Édition électronique

URL : http://journals.openedition.org/abpo/3048

DOI : $10.4000 /$ abpo.3048

ISBN : 978-2-7535-4275-4

ISSN : $2108-6443$

\section{Éditeur}

Presses universitaires de Rennes

Édition imprimée

Date de publication : 30 juin 2015

Pagination : 7-41

ISBN : 978-2-7535-4273-0

ISSN : 0399-0826

\section{Référence électronique}

Marjolaine Lémeillat, « Pierre, fils de Jean II, duc de Bretagne ou l'existence mouvementée d'un noble cadet dissipateur », Annales de Bretagne et des Pays de l'Ouest [En ligne], 122-2 | 2015, mis en ligne le 30 juin 2017, consulté le 19 avril 2019. URL : http://journals.openedition.org/abpo/3048; DOI : 10.4000/ abpo.3048 


\title{
Pierre, fils de Jean II, duc de Bretagne ou l'existence mouvementée d'un noble cadet dissipateur
}

\author{
Marjolaine LÉMEILLAT \\ Doctorante en histoire médiévale (CRBC), \\ ATER à l'université de Bretagne Occidentale
}

"C'estoit un homme qui avoit succédé au dernier Hervé, vicomte de Léon dans ses profusions sans bornes, comme dans ses terres."

Dom LoBINEAU, Histoire de Bretagne, t. I, p. 280.

Cette observation résume bien qui fut Pierre de Bretagne, fils cadet du duc Jean II (1286-1305) ${ }^{1}$. Il est associé dès 1275 par son grand-père Jean I ${ }^{\mathrm{er}}$ (1237-1286) à l'acquisition d'une des très nombreuses cessions que fit Hervé IV de Léon de son patrimoine; mais c'est surtout à partir de 1291 qu'il se fait remarquer, lorsqu'il se retrouve retenu à Paris pour 9000 livres de dettes. Ce scandale lui vaut l'attention de dom Lobineau, puis de dom Morice, frappés par l'ampleur de la chose. Toutefois, mise à part cette affaire, les Mauristes ne lui consacrent que quelques courts passages dans leurs publications respectives ${ }^{2}$. Arthur de La Borderie le gratifie d'une courte notice dans son " Recueil d'actes ${ }^{3}$ ", mais Pierre n'apparaît nulle part dans son Histoire de Bretagne. Des travaux plus récents ont pu en refaire mention $^{4}$; pour autant, le personnage en lui-même n'a jamais fait l'objet d'une étude approfondie, bien que les sources ne manquent pas.

1. Mes remerciements vont ici à Yves COATIVY pour ses observations sur ce cadet ducal haut en couleur.

2. LoBINEAU, Guy Alexis dom, Histoire de Bretagne, t. I, Paris, Clousier, 1707, p. 280 et 296; Morice, Pierre Hyacinthe, dom, Histoire ecclésiastique et civile de Bretagne, t. I, Paris, Osmont, 1742, p. 204, 212 et 229.

3. La Borderie, Arthur Le Moyne de, "Recueil d'actes inédits des ducs et princes de Bretagne ( $\mathrm{XI}^{\mathrm{e}}, \mathrm{xII}^{\mathrm{e}}, \mathrm{xIII}{ }^{\mathrm{e}}$ siècles) ", Bulletin et mémoires de la Société archéologique d'Ille-etVilaine, t. XVII, 1885 , p. 229 , n. 2.

4. Notamment ceux de GAONACH, Marie, Jean II, duc de Bretagne, 1286-1305, mémoire de maîtrise, université de Bretagne occidentale, Brest, 2009, dact., p. 83 et KERNEVEZ, Patrick, Vicomtes et seigneurs de Léon $d u X I^{e}$ au début $d u X V I^{e}$ siècle, thèse d'histoire médiévale, université de Bretagne Occidentale, Brest, 2011, dact., p. 188. 


\section{Pierre, fils de Jean, duc de Bretagne et comte de Richemont : quelles sources?}

Les sources à son sujet sont essentiellement les mandements de payer et quittances, émis lors du règlement de la succession de Jean II, dont la majeure partie entre 1306 et $1308^{5}$. Sur les 290 actes relatifs à cette succession et conservés aux Archives départementales de Loire-Atlantique sous les cotes E 21 à E 23, 49 concernent les dettes de Pierre de Bretagne ou les dégâts causés par lui (soit $16 \%$ de ce corpus). Sur ces 49 documents, 39 sont rédigés en français et ont fait l'objet d'une transcription par Marie Gaonach dans son mémoire de maîtrise portant sur Jean II, duc de Bretagne $(1286-1305)^{6}$. Les 10 autres actes sont en latin et sont demeurés jusqu'ici inédits, aussi les ai-je inclus en annexe 2 de cet article, à titre de pièces justificatives, accompagnés d'une traduction.

À ceci s'ajoutent 9 actes, également conservés aux Archives départementales de Loire-Atlantique; ils ont fait l'objet d'une publication par dom Lobineau, puis dom Morice, pour 2 d'entre eux et par Arthur de La Borderie, pour 7 autres ${ }^{7}$. Outre cet ensemble de 52 pièces, j'ai pu relever quelques mentions éparses dans les comptes ducaux rendus à Muzillac en 1300 et $1303^{8}$. L'ensemble permet d'apporter un certain éclairage sur les activités de Pierre de Bretagne ${ }^{9}$.

5. Trois actes sont antérieurs (10 octobre et 31 décembre 1290, 19 avril 1305). S'ils ont été inclus dans les actes concernant la succession de Jean II, c'est que pour au moins deux d'entre eux, le litige financier concerné n'a été réglé qu'en 1307.

6. Les actes de Pierre de Bretagne y sont publiés aux p. 173-189.

7. Dom Lobineau, Histoire de Bretagne, t. II, Paris, Clousier, 1707, col. 435-436 et 444-445. Dom Morice, Mémoires pour servir de preuves à l'histoire ecclésiastique et civile de Bretagne, Paris, Osmont, 1742, col. 1107-1127-1128. LA BorDERIE, Arthur de, "Recueil d'actes... ", loc. cit., p. 227, $\mathrm{n}^{\circ}$ CLIII, p. 230-231, n CLIV, p. 236-237, $\mathrm{n}^{\circ}$ CLVIII, p. 240-247, $\mathrm{n}^{\circ}$ CLX-CLXIII.

8. LA BORDERIE, Arthur de, "Supplément aux actes inédits des ducs de Bretagne et de leur gouvernement (XIII ${ }^{\mathrm{e}}$ et XIV ${ }^{\mathrm{e}}$ siècles)", Bulletin et mémoires de la Société archéologique d'Ille-et-Vilaine, t. XXI, 1892, respectivement p. 164-175 et 178-193.

9. En revanche, je n'ai pas inclus l'acte d'échange entre Hervé du Chastel et Hervé de Penhoet, datant de 1282 (dom Morice, Preuves..., op. cit., col. 1063-1064). En effet, je ne partage pas entièrement la suggestion émise il y a quelques années par André-Yves BouRGES, suivant laquelle le personnage qui a apposé son sceau à l'acte aurait peut-être été Pierre de Bretagne ("Les origines fabuleuses de la famille du Chastel ", dans Le Trémazan des du Chastel : du château fort à la ruine, Brest-Landunvez, CRBC/Association SOS Château de Trémazan, 2006, p. 34), et reprise par Patrick Kernevez, Vicomtes et seigneurs de Léon..., op. cit., t. 1, p. 188. Leur argumentation repose sur le fait que l'acte était, selon dom Morice, " scellé aux armes du duc Pierre Mauclerc ", ce qui est évidemment une erreur pour un acte datant de 1282; Pierre de Dreux n'est plus duc de Bretagne depuis 1237 et par ailleurs décédé depuis 1250. Aussi André-Yves Bourges estimait-il qu'une confusion avait dû être faite avec Pierre de Bretagne. Toutefois, si ce dernier a bien repris pour armes un écu échiqueté de Dreux au franc-quartier d'hermines, comme son arrière-grand-père le duc Pierre, il n'est pas le seul dans ce cas : Jean ${ }^{\mathrm{er}}$, puis son fils Jean II après lui reprennent tous deux les mêmes armes. En 1282, le personnage susceptible d'apposer son sceau à cet acte aurait tout aussi bien pu être Jean ${ }^{\text {er }}$, certainement plus intéressé aux affaires du Léon que son petit-fils, qui y est seulement détenteur de coutumes et de péages. 
Vingt de ces actes ont été émis par Pierre lui-même (19 en français et un en latin). Ce sont, je l'ai dit, des reconnaissances de dettes, des mandements de payer ou des quittances. Ils présentent tous, de ce fait, le même profil $^{10}$ : scellés sur simple queue de parchemin, dont les dimensions sont comprises entre un maximum de $255 \mathrm{~mm}$ et un minimum de $165 \mathrm{~mm}$, pour la largeur, et un maximum de $105 \mathrm{~mm}$ et un minimum de $40 \mathrm{~mm}$, pour la hauteur ${ }^{11}$. Les 32 autres actes le concernant ont été émis par une quarantaine de personnes différentes. Parmi les plus notables, sont à relever le roi de France Philippe IV (1285-1314), ainsi que les exécuteurs testamentaires du duc Jean II; les personnes restantes sont toutes des créanciers ou des victimes de Pierre.

Ce dernier a quasiment constamment porté le titre de fils du duc de Bretagne. Cela est naturel quand il est encore mineur : la titulature des enfants rappelle traditionnellement qui est leur père, et éventuellement leur mère. Pierre est donc tout d'abord désigné comme "fiuz a noble homme monsour Jahan de Bretayngne, conte de Rychemont ${ }^{12}$ ", ou " fiuz Monseignor Jahan de Bretayngne ${ }^{13}$ ". Cette titulature connaît une légère variante avec l'accession de Jean II au trône ducal : en 1290, Pierre est ainsi devenu " fiz noble prince Jahein, duc de Bretangne, conte de Richemont ${ }^{14}$ ".

Après le décès de ce dernier, il s'intitule d'abord simplement " Pierre de Bretagne ${ }^{15}$ ". Il ne précise qu'une seule fois qu'il est chevalier, en $1306^{16}$. Dans les six autres actes qu'il a émis en 1306, il rappelle sa filiation avec Jean II, de la façon suivante : "Pierres, jadis fiulz dou duc de Bretaigne et conte de Richemond ${ }^{17}$. " J'aurais tendance à attribuer ce souci de précision à la volonté de Pierre de rappeler, dans le cadre du paiement de ses dettes, quelle est sa filiation. En effet, ce sont les exécuteurs testamentaires de son père qui pourvoient à ces versements divers et variés. Il ne juge sans doute pas inutile d'insister sur le fait que le défunt duc était son père, ce qui justifie, d'une certaine manière, le paiement de ses dettes lors du règlement plus général de la succession.

Quant à ses créanciers, ou aux exécuteurs testamentaires ducaux, ils l'appellent presque tous " monsour Pierres de Bretaigne ", pour les actes

10. À une exception près, pour l'acte du 21 mai 1291 (Arch. dép. de Loire-Atlantique, E 209, $\mathrm{n}^{\circ}$ 21). Il se distingue par un scellage en double queue et par de grandes dimensions : larg. $190 \mathrm{~mm} \times$ haut. $250 / 260 \mathrm{~mm}$ (dont $20 \mathrm{~mm}$ de repli).

11. A été exclu de cette étude un acte coupé en deux, dont la largeur se trouve, de ce fait, complètement tronquée (Arch. dép. de Loire-Atlantique, E 21, $\mathrm{n}^{\circ} 14$ ).

12. LA BORDERIE, Arthur de, "Recueil d'actes... ", loc. cit., p. 227, ${ }^{\circ}$ CLIII et p. $231, \mathrm{n}^{\circ}$ cLIV.

13. Ibidem, p. $237, \mathrm{n}^{\circ}$ CLVIII.

14. GaONACH, Marie, Jean II..., op. cit., p. 173.

15. Cette formulation revient huit fois entre le 19 avril 1305 et le 10 mars 1308 , tout en sachant que deux actes n'apportent aucune réelle indication, étant donné qu'ils ont été endommagés et que la titulature a pu en être partiellement tronquée (GAONACH, Marie, Jean II..., op. cit., p. 114 pour l'acte du 13 janvier 1306 n. st. et p. 185, pour l'acte du 19 mars 1307).

16. GaONACH, Marie, Jean II..., op. cit., p. 180.

17. Ibidem, p. 176. 
en français. Dans les dix textes en latin, il est appellé " dominus Petrus de Britannia $^{18}$ " (le seigneur Pierre de Bretagne), et dans cinq cas, l'expression est précédée de la mention " nobilis vir ${ }^{19}$ " (noble homme); par deux fois, Pierre est qualifié de " miles $^{20}$ " (chevalier) ${ }^{21}$. Enfin, dans six des documents du corpus, les auteurs ont pris soin de rappeler que leur débiteur est le fils du défunt duc de Bretagne (" filius dicti domini ducis", fils du dit seigneur duc) ${ }^{22}$.

"Monsour Pierres de Bretaigne " dispose par ailleurs de son propre sceau $^{23}$. La plus ancienne attestation remonte à 1291 : il parle alors des " lettres seellées de nostre propre seel ${ }^{24}$ ". " Nostre seel " ou " nostre propre seel " est ensuite la formule qui revient le plus couramment. Outre ce sceau personnel, Pierre semble avoir eu un grand sceau, qui n'apparaît qu'une fois, le 19 mars 1307, comme le " seaul grant dont l'an use an noz contraz ${ }^{25}$ ".

Cinq sceaux différents ont pu être identifiés. Tous sont de forme ronde et présentent les armes de Pierre : un écu échiqueté de Dreux au franc-quartier d'hermines, avec bordure de gueule besantée ${ }^{26}$. Tous comportent visiblement la même titulature, pour autant que l'on puisse encore la déchiffrer suivant les sceaux :

\section{+ S[IGILLUM $]$ PETRI DE BRITANNIA MILITIS}

(Sceau de Pierre de Bretagne, chevalier)

Ce sont ensuite la taille et les ornementations des sceaux qui varient ${ }^{27}$.

18. Cette formulation, dont les terminaisons varient suivant les déclinaisons, se retrouve dans les pièces justificatives $\mathrm{n}^{\circ} 2,3,5,6$ et 8-10 publiées en annexe.

19 . Voir annexe 2 , pièce $\mathrm{n}^{\circ} 5$.

20. Voir annexe 2 , pièces $n^{\circ} 2$ et 5 .

21. Ce sont l'official de Rennes et l'archidiacre de Porhoët qui le gratifient d'une telle précision, mais cela n'est pas systématique, puisqu'étant respectivement auteurs de quatre et trois autres actes, c'est la seule fois où ils qualifient ainsi Pierre de Bretagne.

22 . Voir annexe 2 , pièces $n^{\circ} 8$ et 9 .

23. Ses sceaux reprennent cette titulature. Il en subsiste 19, tous conservés aux Archives départementales de Loire-Atlantique et tous appendus à des promesses ou des mandements de payer un créancier, émis entre le 31 décembre 1290 et le 23 mars 1308. La majorité (15) est en cire verte, trois étant en cire brune et un en cire jaune. Cinq d'entre eux sont à l'état de fragments et deux sont effacés; néanmoins, le corpus restant était suffisant pour rendre possible une étude des sceaux utilisés par Pierre.

24. LA Borderie, Arthur de, "Recueil d'actes... ", loc. cit., p. 242, n CLX.

25. Arch. dép. de Loire-Atlantique, E 22, n 106.

26. Pour être tout à fait exacte, il est évidemment impossible, à partir d'un sceau de cire verte, jaune ou brune, de déterminer la couleur de la bordure. Toutefois, celle-ci était rouge sur les armes de Pierre de Dreux (1213-1237). Il semble peu probable que cette couleur ait été modifiée par ses descendants.

27. Le premier type identifié porte sur deux sceaux, appendus à des actes en date du 31 décembre 1290 et du 29 mai 1291. Ce type mesure $25 \mathrm{~mm}$ de diamètre et présente les armes de Pierre simplement entourées de sa titulature. Pierre a ensuite fait un ajout. Ce deuxième type concerne 9 sceaux, appendus à des actes émis entre 1306 et jusqu'au 16 janvier 1307. Ils sont légèrement plus grands, avec $30 \mathrm{~mm}$ de diamètre, et présentent les armes du sigillant désormais entourées d'un polylobe. Cette même année 1306, Pierre a fait usage d'un autre genre de sceau que le précédent. Le polylobe a été remplacé par 


\section{Dettes, chevaux, vins, " injures et autres mals " : une turbulente existence}

Né en 1269, Pierre est le cinquième et avant-dernier enfant de Jean, comte de Richemont (et futur duc Jean II de Bretagne) et de Béatrix d'Angleterre $^{28}$. Aucun autre élément ne permet de savoir ce que fut son enfance ou l'éducation dont il a pu bénéficier. Sa première apparition officielle correspond à son acquisition de péages et coutumes dans la vicomté de Léon, ce qui fut, à n'en pas douter, une initiative de Jean I ${ }^{\text {er }}$ lui-même, d'autant que son petit-fils est alors âgé de six ans.

En août 1275, Hervé de Léon commence par céder en gage à Pierre de Bretagne l'ensemble de ce qu'il possède sur les péages et coutumes de Saint-Mathieu de Fineterre, et ce pour 4160 livres de monnaie courante ${ }^{29}$; seules sont exclues de cette cession les cessions antérieurement effectuées au profit du duc Jean ${ }^{\mathrm{er}}$, de Guillaume Rimou, bourgeois de Lamballe, et d'Evrart de Nanteuil, citoyen de Paris ${ }^{30}$. Les garanties prises par Hervé de Léon envers Pierre de Bretagne sont classiques : il s'engage à lui garantir l'objet de la transaction, à lui, ainsi qu'à ses héritiers, et s'y oblige pour cela, tant lui que ses héritiers, sur ses biens meubles et immeubles ${ }^{31}$. S'il ne pouvait tenir cet engagement, le duc de Bretagne serait en droit de procéder à une saisie des dits biens, sans délai ni semonce préalable, jusqu'à acquittement de la garantie due ${ }^{32}$. Il est en outre stipulé que Pierre de Bretagne

un quadrilobe sur carré sur pointe, les dimensions de ce troisième type de sceau restant cependant les mêmes, soit $30 \mathrm{~mm}$ de diamètre. Le quatrième type de sceau est le fameux " seaul grant dont l'an use an noz contraz ", dont il ne reste qu'un seul exemplaire et qui est lui aussi aux armes avec quadrilobe sur carré sur pointe, mais ce dernier présente la particularité d'être chargé de fleur de lys (deux au moins sont encore visibles). Ce sceau est par ailleurs le plus grand de tous, avec $40 \mathrm{~mm}$ de diamètre. Enfin, le dernier sceau utilisé par Pierre, pour le dernier acte émis par lui et ayant été conservé, est un sceau de $30 \mathrm{~mm}$ de diamètre avec écu aux armes entouré d'un hexalobe (acte du 23 mars 1308).

28. Jean II et son épouse Béatrix ont en effet eu six enfants : Arthur (le futur Arthur II), Jean, Blanche, Marie, Pierre et Aliénor (GaOnAcH, Marie, Jean II..., op. cit., p. 19).

29. LA BorDERIE, Arthur de, "Recueil d'actes... ", loc. cit., p. 227, n CLIII. " quanque nous avons e poons avoier par queuque reson de droiture, de propriété, de possession e de seyngnorie ès payages e ès costumes de Seint Mahé de Fine Posterne, de l'evesché de Léon, pour quatre mile e huit vinz livres de la corante monoie".

30. Ce même mois d'août 1275, Hervé IV de Léon leur avait vendu, pour dix-sept ans, " les fruiz e les essues de la costume e dou peage de Seint Mahé de Fine Posterne, de l'evesque de Leon, o totes justices et seigneuries e o quanque nos i avions e poions aver ". Le montant de la transaction n'est pas spécifié dans l'acte (dom Morice, Preuves..., t. I, op. cit., col. 1037).

31. "E somes tenuz à garantir ledit Pierres e ses ayers e ceus qui cause auront de lui sus les choses davant dites de touz e encontre touz et meymement de tous douères. $\mathrm{E}$ à cestes garantye faire nous obligeons nous e nos ayers e touz noz biens meubles e non meubles, presenz et futurs, en queuque leu qui is soient " (LA BORDERIE, Arthur de, "Recueil d'actes... ", loc. cit., p. 227-228, n CLIII).

32. «E volons e otréons e requerons le conte de Bretayngne, si il avenoit que nous deffaillissons de garantir ledit Pierres ou ses ayers [...] que le davant dit conte preyge e sesisse e explete de nos biens meubles e non meubles, en queque leu qui is soient, sanz fuite e sanz deloie e sans semonsse e sans sequestre que l'an nous face, e sanz content 
ou ses héritiers sont en droit de vendre les biens cédés, sans qu'Hervé lui-même ne puisse s'y opposer ${ }^{33}$.

Ce premier engagement devient une vente en bonne et due forme, elle aussi conclue au mois d'août 1275, toujours pour la même somme de 4160 livres $^{34}$; les diverses clauses restent les mêmes que dans l'acte précédent. Outre l'âge de l'acquéreur et le fait que le duc Jean $\mathrm{I}^{\mathrm{er}}$ ait déjà eu recours à des membres de sa famille pour conclure des transactions à sa place ${ }^{35}$, un autre élément incite à voir dans cette vente l'intervention ducale : il s'agit de la précision suivant laquelle "ledit Piere paya ${ }^{36}$ ". À 6 ans, il est plus que douteux qu'il ait disposé de 4160 livres à verser à Hervé de Léon. Dans cette affaire, il sert très clairement d'homme de paille : Jean $\mathrm{I}^{\mathrm{er}}$ utilise ce biais pour s'emparer à nouveau de biens dilapidés par Hervé de Léon ${ }^{37}$. Il en profite également avec, sans doute, une autre arrière-pensée : doter son troisième petit-fils d'une terre. En effet, Arthur, l'aîné de ses petits-fils, est destiné à hériter de la couronne ducale; il était sans doute d'ores et déjà prévu que Jean, deuxième petit-fils, serait doté du comté de Richemont (il est à remarquer que c'est Pierre, et non Jean, qui a été choisi comme acquéreur des coutumes et péages de Saint-Mathieu); Pierre, quant à lui, a été pourvu de biens issus de la vicomté de Léon. Toutefois, contrairement à ce qui est parfois encore écrit, il n'en a jamais été nommément vicomte.

Une fois l'ensemble des biens d'Hervé de Léon récupérés ${ }^{38}$, Jean $\mathrm{I}^{\mathrm{er}}$ en obtient ratification par Roland de Dinan et Amice, son épouse, respective-

que nous i puissons metre " (LA BORDERIE, Arthur de, "Recueil d'actes... ", loc. cit., p. 228, $\mathrm{n}^{\circ}$ CLIII).

33. " nous volons e gréons e otréons que ledit Pierres ou ses ayers, ou ceus qui cause auront de lui, puissent metre en vante les davant dites choses quant i voudron e i lor plera, sanz content et sanz debat que nous i puissens metre, ne par nous ne par autres " (LA Borderie, Arthur de, "Recueil d'actes... ", loc. cit., p. 228, n CLIII).

34. " nous avons vendu à Pierres de Bretaygne, fiuz à noble homme Monsour Jahan de Bretaygne, conte de Richemont, audit Pierres et à ses aiers à fiu eritage ou à ceus qui cause auront de lui, à avoier et à possaier en pès quanque nous avons e poons avoier par queque reson, de droiture, de propriété, de possession e de seyngnorie à Seint Mahé ès costumes e ès payages de Seint Mahé de Fine Posterne de l'evesché de Léon, pour quatre mile e huit vinz livres de la corante monaie " (LA BORDERIE, Arthur de, " Recueil d'actes... ", loc. cit., p. $230-231, \mathrm{n}^{\circ}$ CLIV).

35. Le principal exemple en la matière étant Pierre de Bretagne, son fils puîné (LÉMEILLAT, Marjolaine, "Jean I ${ }^{\mathrm{er}}$, duc de Bretagne, comte de Richemont et Pierre de Bretagne, chevalier, son fils ", dans Jean-Christophe Cassard, historien de la Bretagne, COATIVY, Yves, Gallice, Alain, HERY, Laurent et LE PAGE, Dominique (dir.), Morlaix, Skol Vreizh, 2014, p. 337-345).

36. La BorDERIE, Arthur de, "Recueil d'actes... ", loc. cit., p. 231, n CLIV.

37. Toujours en cette année décidément faste de 1275 , Jean $\mathrm{I}^{\mathrm{er}}$ obtient notamment le port de Saint-Mathieu et les coutumes des navires qu'y percevait Hervé de Léon. Ce dernier les lui cède : "ego dedi et concessi, do etiam et concedo domino meo Johanni, duci Britannie quicquid juris, proprietatis, possessionis et dominii habebam et habere poteram quacumque de causa in portu Sancti Mathei de Finibus Terrarum et in costumis navium racione dicti portus " (LA BORDERIE, Arthur de, "Recueil d'actes... ", loc. cit., p. 231-232, n clv).

38. L'ensemble des cessions, entre 1239 et 1276, a été publié par Arthur de LA BorDERIE, "Recueil d'actes... ", loc. cit., p. 217-237, nº cXLVII-CLVIII. 
ment beau-frère et sœur du dit Hervé : ceux-ci incluent dans leur acte les acquisitions de Pierre de Bretagne ${ }^{39}$. De même, le 7 juin 1298, lorsqu'Ame, fille d'Hervé de Léon, et son époux, Prigent de Tonquédec, abandonnent leurs revendications sur l'héritage de Léon, ils retirent toute plainte entre autres contre Pierre de Bretagne ${ }^{40}$.

Pierre est à nouveau mentionné en 1279 , dans une concession de Jean I $^{\text {er }}$ en faveur de marchands de Bayonne, auxquels il cède la sécherie de SaintMathieu-de-Fineterre que ceux-ci possédaient auparavant des vicomtes de Léon, et ajoute les droits et appartenances y afférant, pour 130 livres annuelles de monnaie courante de Bretagne. En échange de quoi, il s'engage à protéger les marchands et leurs héritiers " contre tous nos héritiers, et surtout contre Pierre de Bretagne, et n'importe quels autres, au sujet de la sécherie susdite ${ }^{41}$ ". Pierre est effectivement concerné au premier chef, puisqu'il possède des biens dans la région immédiate.

Une petite dizaine d'années plus tard, il réapparaît dans les sources en 1291, en prison et surendetté : le 29 mai, il indique être retenu sous serment à Paris, pour 9000 livres de dettes, dues à des prêts et achats de chevaux ${ }^{42}$. Cette déplaisante affaire est mal venue ${ }^{43}$ : Jean II s'empresse d'intervenir en commençant par faire sortir son fils de prison, en s'engageant pour lui à rembourser la somme due. Ses débiteurs acceptent dès lors la délivrance

39. «Sachent touz que les achaz, les conquestes e les obligacions que nostre cher seignor Jahan, duc de Bretaigne, Pierres fiuz Monseignor Jahan de Bretaigne, et Guillaume de Kemperelé ont fez o le visconte de Léon, nostre frere nous avons fermes e estables " (LA BORDERIE, Arthur de, "Recueil d'actes... ", loc. cit., p. 236-237, n CLVIII).

40. " feimes pès e accort o nostre tres chier seignour, tres noble prince Jahan, duc de Bretaigne, conte de Rychemont, e o les exequtours dou testament son pere desus dit de toutes les choses dessus dites et de toutes les demandes e reisons e accions que nous diz Prigent e Ame peussons fere e demander par quelque maniere e par quelque reison de tout le temps passé audit nostre chier seignour le duc de Bretaigne desus dit, ne à ses heirs, à monsor Pierres de Bretaigne, filz audit duc, e aux exequtours dou testament au père audit duc desus diz " (LA BoRDERIE, Arthur de, "Recueil d'actes... ", loc. cit., p. 246, $\mathrm{n}^{\circ}$ CLXIII).

41. «Pro quibus sex viginti et decem libris annuis, tenemur et promittimus ipsos mercatores, heredes, successores et suos nuntios ad hoc missos garentizare in perpetuum et deffendere contra omnes heredes nostros et maxime contra Petrum de Britannia et quoscunque alios, super siccaria antedicta " (LEMEILLAT, Marjolaine, Les actes de Jean I ${ }^{\text {, }}$, duc de Bretagne (1237-1286), Rennes, PUR/Société d'Histoire et d'Archéologie de Bretagne, 2014, p. 280$283, \mathrm{n}^{\circ} 160$, acte du 10 mai 1279).

42. "nous fussons tenuz et obligiez par lettres et par serement de fere et tenir prison et ostage en la vile de Paris pour nuef mile livres de tournois petiz, que nous deviens de cause de prest et de chevaus achatez à Hevrart de Seint Venant le joune et au Borgne de Douoi et à Pierres de Quamnesi, marchans de chevaus, lesquiex chevaus lesditz marchaans nous baillerent à nous et à nostre commandement " (LA BORDERIE, Arthur de, "Recueil d'actes..." "loc. cit., p. 241, n CLX).

43. Dom LOBINEAU remarque au passage que cette affaire intervient juste un an avant le mariage d'Alix, sœur de Pierre, avec Guy de Châtillon. Il est envisageable que les deux familles étaient alors déjà en négociation matrimoniale. Les frasques du futur beau-frère de Guy de Châtillon tombent au mauvais moment (Histoire de Bretagne..., t. I, op. cit., p. 280). 
de Pierre ${ }^{44}$. Toutefois, " nostre cher pere " n'entend pas tenir son fils quitte pour cela : celui-ci est tenu de le rembourser ${ }^{45}$. Par ailleurs, le duc s'inquiète surtout du sort des biens que possède l'enfant prodigue : pourrait-il un jour en venir à les dilapider pour s'acquitter de ses dettes?

Aussi Jean II pare-t-il à cette éventualité : son fils s'engage par écrit à lui rendre l'argent pour lequel il s'est porté caution, mais il s'y oblige sur ses biens : " quamque nous avons et avoir poons par quelque maniere de droit et de fet et de saisine en la duché de Bretaigne, et especialment quamque nous avons et avoir poons en la visconté de Léon en Bretaigne ${ }^{46}$ ". Pierre ajoute également, concernant la vicomté de Léon : " Et prometons audit nostre cher pere que de la viconté de Leon desus dite o nule autre persone que o lui nous ne ferons convenance ne aliance outre sa volanté; et se aucunes en fesions, que ja ne soit, nous voulons que ele soient nules et de nule vertu ${ }^{47}$. " L'enjeu est manifestement trop important pour que l'on puisse laisser Pierre de Bretagne agir de manière irresponsable. Celui-ci doit en outre se présenter le lendemain devant Philippe le Bel, qui, d'une part, confirme le texte dont il donne vidimus, et d'autre part, reçoit le serment de Pierre de respecter ce qui y est écrit ${ }^{48}$.

L'affaire n'en reste pas là. Jean II, bien qu'aucun élément n'en fournisse la preuve, a certainement dû s'acquitter des dettes de son fils; il n'a pas oublié non plus de récupérer les biens de ce dernier. Son émancipation est définitivement prononcée en présence de Philippe le Bel, en octobre 1293; Pierre est naturellement présent, et surtout, " expressément consen$\operatorname{tant}^{49}$ " (en tout cas, officiellement). De plus, il cède à son père " tous ses

44. « nostre cher pere Jehan [...], voulant metre conseil en nostre delivrance de l'ostage et de la prison que nous estions tenuz fere, si comme il est desus dit, à nostre requeste, envers lesdiz marchaans de la summe de l'argent desus dit pour nous se voult lier et obliger, par laquele obligacion nostre cher pere desus dit fist fere envers lesdiz marchaanz nostre delivrance. Lesdiz marchaans nous ont quitez et delivrez de l'ostage et de la prison desus diz, et se vouldrent tenir audit nostre cher pere de la summe de l'argent desus dit " (LA BORDERIE, Arthur de, "Recueil d'actes... ", loc. cit., p. 241, n ClX).

45. "Auquel nostre cher pere desus dit nous recognoissons devoir les nuef mil livres desus dites, et li prometons enterignement lui rendre les deniers desus diz à sa pleine volonté " (LA Borderie, Arthur de, "Recueil d'actes... ", loc. cit., p. 241, n CLX).

46. LA BORDERIE, Arthur de, "Recueil d'actes... ", loc. cit., p. 241, n CLX.

47. Ibidem.

48. "Predictus siquidem Petrus in presentia nostra constitutus premissa et singula recognovit ut superius continentur, ea promittens tenere et complere et non contra facere vel venire, juramento ab eo in manu nostra prestito corporali " (LA BORDERIE, Arthur de, "Recueil d'actes..." "loc. cit., p. $242, \mathrm{n}^{\circ}$ CLXI).

49. Il avait déjà été fait référence à son émancipation dès le 29 mai 1291, lorsque Pierre déclare : "Et connoissons que toutes ces choses desus dites feismes nous jà émancipé solennelment " (LA BORDERIE, Arthur de, "Recueil d'actes... ", loc. cit., p. 241, n CLX). En octobre 1293, cela est bel et bien acté par le roi de France : "dilectus fidelis noster Johannes, comes Britannie, posuit extra patriam potestatem et emancipavit Petrum de Britannia, filium suum, presentem et expresse consentientem " (dom MoricE, Preuves..., t. I, op. cit., col. 1107). Il est à remarquer que Pierre de Bretagne est émancipé tardivement : il a en effet 24 ans en 1293. Cela laisse à penser que sa tendance à la dépense avait déjà dû 
droits seigneuriaux, possessions, propriétés, juridictions, pouvoir seul et mêlé et tout autre droit qu'il a et peut avoir dans la vicomté de Léon, sise dans le comté de Bretagne, et dans toutes les dépendances médiates ou immédiates de cette vicomté ${ }^{50}$ ". Ladite vente est confirmée par le roi de France en février 1294. Comme dans l'acte précédent, il est rappelé, de manière classique, que Pierre a pris sa décision spontanément, sans pression aucune ${ }^{51}$ (ce qui est tout de même loin d'être assuré) et qu'il cède l'ensemble de ce qu'il possède dans la vicomté de Léon pour 14000 livres tournois ${ }^{52}$. C'est un prix important et bien supérieur à ce qu'avaient coûté les coutumes et péages de Saint-Mathieu, acquis pour 4160 livres. Il est possible que Pierre de Bretagne ait possédé d'autres biens ou redevances dans la vicomté, mais sans doute pas au point de justifier un ajout de plus de 9000 livres. Il semble plus probable que, sous couvert de cette transaction, Jean II verse à son fils le prix de la vente, assortie d'un supplément destiné à éponger d'autres dettes, restées ignorées faute de sources.

Celles-ci renseignent davantage sur ses déplacements. Pierre de Bretagne voyageait apparemment volontiers. Présent à Paris en 1291, il y est resté, plus ou moins contraint et forcé jusqu'en février 1294, le temps que son père satisfasse ses créanciers. Vassal obéissant, il est présent à la bataille de Courtrai, le 11 juillet 1302, puis revient au camp d'Arras lors de la convocation royale de $1303^{53}$. Plusieurs quittances laissent également entrevoir qu'il se déplaçait aussi beaucoup dans le duché de Bretagne ${ }^{54}$. Il s'est notamment distingué dans le diocèse de Rennes, commettant des pillages dans la paroisse de Groys, ainsi qu'à Saint-Aubin-du-Cormier ${ }^{55}$; il a dû très probablement séjourner à crédit dans la ville même de Rennes, le temps d'y contracter 600 livres de dette en faible monnaie auprès de commerçants de bouche ${ }^{56}$. Sans surprise, il avait visiblement ses habitudes

être remarquée; et sans doute a-t-il été maintenu sous tutelle afin de limiter les dégâts, et ce, jusqu'à ce que la situation devienne intenable.

50. « cessit, concessit et transtulit quicquid juris domini, possessionis et proprietatis, jurisdicionis, imperii meri et mixti et cujuscumque alterius juris habet et habere potest in vicecomitatu Leonie, sito in comitatu Britannie, in ejusque vicecomitatus pertinentiis omnibus mediatis vel immediatis" (dom Morice, Preuves..., t. I, op. cit., col. 1107).

51. "non vi, non metu nec dolo ad hoc inductus " (LA BORDERIE, Arthur de, "Recueil d'actes..." "loc. cit., p. 243, n ${ }^{\circ}$ CLXII).

52. " quicquid juris, dominii, proprietatis et possessionis, juridicionis, imperii meri et mixti, et cujuscumque alterius juris habebat seu habere poterat in vicecomitatu Leonense, sito in comitatu Britannie, et in ejusdem vicecomitatus pertinentiis omnibus, medietatis et immediatis, pro pretio quatuordecim milium librarum turonensium " (LA BORDERIE, Arthur de, "Recueil d'actes..." ", loc. cit., p. 244, n CLXII).

53. Voir l'itinéraire en annexe.

54. La date de ces déplacements reste toutefois impossible à déterminer : la plupart d'entre eux se situent avant 1305 (avant le décès de son père, dont la protection en faveur de son puîné est souvent mentionnée par les plaignants).

55 . Voir annexe 2 , pièces $n^{\circ} 7$ et 10 .

56. Il doit 400 livres de faible monnaie aux panetiers de Rennes et des environs, et 200 livres de la même monnaie aux poissonniers et bouchers de la ville (GAONACH, Marie, Jean II..., op. cit., p. 185). 
dans les demeures paternelles, à Benester ou Suscinio ${ }^{57}$. Enfin, durant les années 1306-1307, il est surtout présent aux sessions de dédommagement prévues dans le cadre de la succession de Jean II, soit le plus souvent à Ploëmel et Quimperlé ${ }^{58}$.

Les remboursements de ses dettes renseignent aussi sur ses goûts et distractions. Les chevaux sont visiblement sa passion première. Il doit entre autres à l'achat d'équidés les 9000 livres de dettes qui lui valent la prison en $1291^{59}$. Vue cette somme, il faut en conclure que, soit ses autres emprunts étaient d'un montant très élevé, soit il a acheté des bêtes très onéreuses. L'acquisition de montures revient à deux autres reprises. Sa dette envers Richard de Maure provient pour moitié de l'achat d'un cheval $^{60}$; le 15 avril 1306, il reconnaît devoir à Guillaume Le Sergent 11 livres, pour un cheval, qui d'ailleurs, dans ce cas précis, n'est pas pour lui ${ }^{61}$. Il doit également 10 livres de faible monnaie pour le cheval de Jean Le Clerc $^{62}$.

Le vin, d'Anjou et de Gascogne notamment, est une autre tentation, ainsi qu'en atteste un acte du 9 janvier 1307, rappelant que Guillaume de La Bouteillerie et son épouse Perrone demandent le dédommagement de " quatre pipes de vin d'Anjou, un tonel de vin de Guascoigne, que il disaent que monsour Pierres de Bretaigne e ses gienz avaent euz ${ }^{63}$ "; de même, Jean Dupuis reçoit 12 livres de faible monnaie le 20 mars 1307, pour du vin que lui avaient pris les gens de Pierre de Bretagne ${ }^{64}$. Deux selliers rennais figurent parmi les créanciers de Pierre, pour une somme de 320 livres $^{65}$. Par ailleurs, les comptes ducaux de Muzillac, rendus en 1303, prouvent que Pierre se sert en fonction de ses envies : sont ainsi mentionnés " dous tonneaux de vin de Jarnac et un tonneau de vin de Gascoigne, exceptié ce

57. C'est à Benester qu'il va se réfugier après avoir pris à Guillaume Le Sergent son cheval (voir annexe 2 , pièce $n^{\circ} 9$ ).

58. Voir itinéraire en annexe.

59. " nuef mile livres de tournois petiz, que nous deviens de cause de prest et de chevaus achatez à Hevrart de Seint Venant le joune et au Borgne de Douoi et à Pierres de Quamnesi, marchans de chevaus, lesquiex chevaus lesditz marchaans nous baillerent à nous et à nostre commandement " (LA BORDERIE, Arthur de, "Recueil d'actes... ", loc. cit., p. $\left.241, \mathrm{n}^{\circ} \mathrm{CLX}\right)$.

60. " vint livres de la vente d'un cheval a nous vendu de cleuy clerc e receu " (GAONACH, Marie, Jean II..., op. cit., p. 173).

61. " nous Pierres de Bretaigne devons e semes tenuz a paer a Guillaume Le Sergiant de Guengamp onze libres de la monae corante de la vanzon de un cheval que nous eumes de luy a nostre requeste pour doner a Guillaume Urol " (GAONACH, Marie, Jean II..., op. cit., p. 175).

62. GAONACH, Marie, Jean II..., op. cit., p. 176-177. Ledit Guillaume Urol étant inconnu par ailleurs, il est impossible d'expliquer ce qui lui a valu ce don de la part de Pierre de Bretagne.

63. Arch. dép. de Loire-Atlantique, E 22, n 10.

64 . Voir annexe 2 , pièce $\mathrm{n}^{\circ} 6$.

65. GAONACH, Marie, Jean II..., op. cit., p. 183. 
que monsour Pierre en but " et " 6 tonneaux de vin, persomet 10 pipes que monsour Pierres prist ${ }^{66}$ ".

Pour le reste, les quittances de remboursement ne sont guère disertes. Les débiteurs se contentent d'indiquer le versement de la somme et de s'en déclarer contents, mais ne s'étendent pas sur les causes de la dette. Seules deux quittances le spécifient : le 22 janvier 1307, Mahaut, veuve de Jean Legac, et Peronne, veuve de Guillo Michel, reconnaissent avoir reçu 60 sous de faible monnaie, que Pierre de Bretagne leur devait pour du pain de froment ${ }^{67}$. Le 24 janvier 1307, Pierre Raoul de Penhers obtient 10 livres de faible monnaie; lui-même ne précise pas la cause de ce remboursement, mais il est indiqué en marge du document qu'il est fait pour Pierre de Bretagne, pour deux bœufs ${ }^{68}$ (peut-être ces animaux sont-ils le fruit d'un pillage). Le 19 mars 1307, Pierre de Bretagne déclare que 600 livres de faible monnaie ont été remboursées aux panetiers, bouchers et poissonniers de Rennes, à savoir 400 livres pour les panetiers et 200 livres pour les bouchers et poissonniers. Et encore, tout ce qu'il leur doit n'a pas été payé, les 600 livres sont à déduire de sa dette globale ${ }^{69}$.

Certains plaignants lui reprochent également plusieurs préjudices. Des déprédations transparaissent dans les comptes de dépense de Roland Le Lombard, lors de l'exécution du testament de Jean II. Il verse ainsi 12 livres à Guillaume de Bochel, qui se plaint de dommages à lui causés par Pierre de Bretagne et un dénommé Jean Blanchart ${ }^{70}$. Pierre Le Fevre, clerc, est quant à lui dédommagé de 40 livres " pour toutes ses peticions, injures et autres mals que Mons. Pierre de Bretaigne li fit ». Enfin, Jehan Evesne reçoit 100 sous "pour les torfaiz que mons. Pierre de Bretaigne li avoit faiz ${ }^{71}$ ". Ces comptes ne glosent cependant guère sur la nature exacte des " injures " commises.

Quelques quittances sont plus explicites : Jean Vacher, recteur de l'église de Groys, évoque des " prises, ravages et occupations " au préjudice de ses terres ${ }^{72}$. Eudes Frelon et son père, quant à eux, ont subi le ravage

66. LA BORDERIE, Arthur de, "Supplément aux actes... ", loc. cit., p. 178-193, n xxIII et $\mathrm{n}^{\circ} 1$ et 56 .

67. « sexante souz de la feible moneye que il [Pierre de Bretagne] nous deveyt de vente de pen de forment qui estoit en communauté entre nous " (GAONACH, Marie, Jean II..., op. cit., p. 183).

68 . Voir annexe 2 , pièce $n^{\circ} 4$.

69. " quatre cenz libres de la dite monaie aux panetiers de la ville de Reines et d'anviron, et les autres dous cenz libres au bocherz et au poissonierz de la dite ville de Reines, an amenuyssant de la dette en laquele nous et noz genz en notre nom estions obligiez au diz bolangierz, poissonierz, bocherz " (GAONACH, Marie, Jean II..., op. cit., p. 185).

70. « Guillelmo de Bochellis, pro retornacione dampnorum que sustinuit per negligenciam domini ducis in deffendendo ipsum super ecclesia Beati Johannis desuper Vinoniam contra dominum Petrum de Britannia et Johannem Blanchart presbyterum, 12 l." (LA BORDERIE, Arthur de, «Supplément aux actes...", op. cit., p. 246, acte n ${ }^{\circ}$ xxvi et n ${ }^{\circ} 26$ ).

71. LA BORDERIE, Arthur de, "Supplément aux actes... ", op. cit., p. 248-249 et 259, acte $n^{\circ}$ XXVI et $n^{\circ} 43$ et $n^{\circ} 110$.

72. Voir annexe 2 , pièce $n^{\circ} 2$. 
et le pillage de leurs bois et vignes ${ }^{73}$. Guillaume, recteur de l'église paroissiale de Lécousse, et sa mère ont vu leur maison de Saint-Aubin-du-Cormier fracturée et pillée ${ }^{74}$. Enfin, en date du 9 mai 1308, l'abbé de Boisbilly relate les dommages causés par Pierre de Bretagne au défunt Jean Lenfant, en l'occurrence, une perception abusive de dîme ${ }^{75}$.

Pierre n'agit pas seul lors de ce genre d'expédition : il a sous ses ordres gens et serviteurs, qui se chargent aussi des prises de vin ou des pillages et déprédations pour le compte de leur maître ${ }^{76}$. On a vu ci-dessus le témoignage de Guillaume de La Bouteillerie ou celui de Jean Dupuis qui, tous deux, citent Pierre de Bretagne " e ses gienz ". Il est aussi une fois mention de complices participant aux pillages ${ }^{77}$, peut-être des compagnons de fredaine. Les textes ne fournissant guère plus de détails, il est malaisé de déterminer qui composait exactement le proche entourage de Pierre de Bretagne, qu'il s'agisse de ses serviteurs ou de ses amis. Seuls deux noms apparaissent dans les sources : Jean de Beaumont, qui fut son clerc (auquel il doit d'ailleurs aussi de l'argent ${ }^{78}$; et un dénommé Guillaume Urol, auquel il destinait le cheval acquis aux dépens de Guillaume Le Sergent, mais sur lequel on n'en sait pas plus ${ }^{79}$. En tout cas, le fils du duc de Bretagne semble avoir profité de sa position pour agir comme bon lui semblait, y compris en voyou.

Cela lui était d'autant plus facile que le duc Jean II l'a couvert. Cette attitude est sous-entendue aussi bien dans les quittances elles-mêmes que dans les comptes de Roland Le Lombard : le duc est dit avoir été négligent ou avoir fait preuve de " négligence ", pudique terme trouvé afin de justifier les remboursements dus aux victimes, sans pour autant flétrir la mémoire du défunt duc de Bretagne. Jean II s'est ainsi montré négligent pour Guillaume de Bochel, tout comme pour Jean Lenfant, malgré ses plaintes ${ }^{80}$. Il a été "négligent et indifférent " pour Eudes Frelon et le père de ce dernier ${ }^{81}$. Dans les comptes de Roland Le Lombard se trouve aussi le cas de Pierre Le Fevre,

73. Voir annexe 2 , pièce $n^{\circ} 7$.

74. Voir annexe 2 , pièce $n^{\circ} 10$.

75. " ou temps que Johen Leeffant viveyt, monsour Pierres de Bretangne leva, par l'espaice de oyt anz ou environ, toute sa deeme de l'anné ", ce qui est un "grant tort que monsour li feseit " (GAONACH, Marie, Jean II..., op. cit., p. 188).

76. Jean Vacher, mais aussi Guillaume, recteur de Lécousse, font mention des gens et serviteurs du duc (voir annexe 2 , pièces $\mathrm{n}^{\circ} 2$ et 10 ).

77. C'est Jean Vacher qui apporte cette précision (voir annexe 2, pièce $\mathrm{n}^{\circ} 2$ ).

78. Pierre doit 73 livres à " Jahan de Beaumont, nostre clerc " (Arch. dép. de LoireAtlantique, E 21, $\mathrm{n}^{\circ} 29$ ).

79. GAONACH, Marie, Jean II..., op. cit., p. 175. C'est la seule source où apparaît Guillaume Urol; l'on ignore donc tout de ce personnage.

80. " pro retornacione dampnorum que sustinuit per negligenciam domini ducis in deffendendo ipsum super ecclesia Beati Johannis desuper Vinoniam contra dominum Petrum de Britannia et Johannem Blanchart presbyterum, 12 l. " (LA BORDERIE, Arthur de, "Supplément aux actes...", loc. cit., p. 246, acte $\mathrm{n}^{\circ}$ XxvI et $\mathrm{n}^{\circ} 26$ ). Pour Olivier Lenfant, l'acte indique : " e s'em plegnit le dit Johen par meinte fen a mon segnor de Bretangne, qui mort est, dou grant tort que monsour li feseit e fut troé que mon segnor de Bretangne en fut negligent " (GAONACH, Marie, Jean II..., op. cit., p. 188).

81 . Voir annexe 2 , pièce $n^{\circ} 7$. 
pour lequel il est écrit : "A Pierre Le Fevre, clerc, pour toutes ses peticions, injures et autres mals que Mons. Pierre de Bretaigne li fit, sus lesqueles fut requis Mons. le duc de li faire droite justice, de laquele justice faire ledit duc a esté negligent, pour sa negligence purgier, 40 livres ${ }^{82}$. "Seul Guillaume Le Sergent ne s'embarrasse pas de mots et indique de manière très claire que le duc a refusé de lui rendre son cheval, bien que Pierre le lui ait pris ${ }^{83}$. De fait, ce dernier n'étant plus apanagé depuis son émancipation, il semble bien qu'il soit entretenu par son père et qu'une fois à court d'argent, les quittances soient devenues son mode de paiement, quand il ne laisse pas de dettes ${ }^{84}$. Dans tous les cas, peu lui chaut visiblement, puisque le duc en assume les frais.

En somme, Pierre de Bretagne présente les traits d'un fils de puissant personnage qui se sait intouchable : dépensier et sans vergogne, voyou et sans scrupule. Il délivre assez souvent des lettres en vertu desquelles il s'engage à réparer les dégâts causés; mais il les donne d'autant plus volontiers qu'elles ne l'engagent à rien. Tant qu'il ne se décide pas à payer et tant qu'il est protégé par sa parenté, ses lettres ne sont que parchemin sans valeur. La meilleure preuve en est l'exemple de Richard de Maure, qui attend dix-sept ans le remboursement de ses 40 livres $^{85}$.

\section{De 36 sous à 370 livres : le règlement des dettes}

Pierre de Bretagne est constamment endetté. La première reconnaissance de dettes connue remonte au 10 octobre 1290 : le fils du duc de Bretagne fait savoir qu'il doit 40 livres de monnaie courante au clerc Richard de Maure ${ }^{86}$.

La majeure partie de ses dettes est révélée lors du décès de son père, lorsque les exécuteurs testamentaires de ce dernier s'emploient à payer

82. LA BORDERIE, Arthur de, "Supplément aux actes... ", loc. cit., p. 248-249, acte n ${ }^{\circ}$ XXVI et $\mathrm{n}^{\circ} 43$.

83. Voir annexe 2 , pièce $n^{\circ} 9$.

84. Il accumule en effet les dettes de très petit montant (36 sous et 40 sous de bonne monnaie, ou encore 60 sous de faible monnaie) et auprès de personnes modestes (telles des veuves), ainsi que les impayés, le plus souvent chez des fournisseurs de produits de bouche (panetiers, poissonniers), mais jamais rien de luxueux ou de prestigieux, comme c'est souvent le cas pour les nobles qui vivent à crédit. Je pense que, dans son cas, il ne pense pas tant à vivre suivant son rang qu'à vivre tout court et à satisfaire ses envies avec les moyens qu'il a.

85. Prêtées en 1290 , elles lui sont remboursées en 1308 .

86. "Sachent que toz que nous devon e semes tenuz a Richart de Maure, clerc, en quarante livres de monae corante, rendre a yceluy clerc a sa volente " (GAONACH, Marie, Jean II..., op. cit., p. 173). Ce n'est certainement pas la seule reconnaissance de dette qu'il a dû signer durant les années 1290; mais si celle-ci est connue, c'est sans doute aussi parce que, comme elle a été finalement réglée par les exécuteurs testamentaires de Jean II, les autres actes relatifs à cette dette ont été inclus dans le dossier des quittances du défunt duc, avec lesquelles ils ont été conservés. 
tout ce que doit le fils prodigue. Ceux-ci sont au nombre de treize, chiffre symbolique s'il en est, et répartis comme suit :

- quatre "religious hommes e honestes ${ }^{87}$ ", à savoir Even, abbé de Prières; Geoffroi Broessin, gardien des frères mineurs de Nantes; le gardien des frères mineurs de Vannes,

- quatre " amez et fealz chevaliers ", soit Jean de Maure, le père; Étienne de La Guerche; Robin Raguenel et Geoffroy de Guignen,

- cinq " amez et fealz clercs ", que sont Alain de Châteaugiron, archidiacre de Rennes ; maître Geoffroi d'Anast ; maître Thomas d'Anast ; Jacques de Saint-Loup, chapelain du défunt duc et Aubry de Baudement.

Tous sont des personnes de confiance de Jean II. Plusieurs d'entre eux sont déjà au fait des affaires et surtout du maniement de l'argent et des comptes, tels Robin Raguenel, par ailleurs sénéchal de Rennes, mais aussi Geoffroi de Guignen, Jean de Maure, l'abbé de Prières ou Geoffroi et Thomas d'Anast, ainsi qu'en attestent leur apparition dans les comptes, ou les legs ducaux $^{88}$. Certains d'entre eux ont aussi déjà eu l'occasion de travailler de concert, comme ce fut le cas pour Robin Raguenel et Jean de Maure ${ }^{89}$, mais aussi pour l'abbé de Prières et le gardien des franciscains de Vannes ${ }^{90}$. Enfin, tous connaissent également déjà les fameux Roland Le Lombard et Jean Le Roy, principaux bailleurs des fonds utilisés pour le règlement de la succession ${ }^{91}$.

87. Ainsi que le duc les qualifie dans son testament (Arch. dép. de Loire-Atlantique, E 20, $\left.\mathrm{n}^{\circ} 1\right)$.

88. Robin Raguenel apparaît deux fois dans les comptes rendus à Muzillac en 1300 et 1303, gérant la levée des fermes des pêcheries de Châteaulin, ainsi que la perception des redevances en nature (LA BORDERIE, Arthur de, "Recueil d'actes... ", loc. cit., p. 174, $\mathrm{n}^{\circ}$ 123) : "Robin Raguenel, 18 l., 8 s., 4 d. - Item, il deit lever des fermes des pescheries de Chasteaulyn 60 l. de l'an qui finira ou maeis de may. - Item, ledit Robin deit 104 bricaz [mesure de grain] de seille. - Item, 664 bricaz de aveine. - Item, 258 gelines "; ibid., p. 182 , $\mathrm{n}^{\circ} 32$ : "Robin Raguanel, 17 l. - Item, 4 l. pour Beniat. - Item, 65 barriques de seille et 240 barriques de avenne, 500 gelines ». Il figure aussi p. $170, \mathrm{n}^{\circ} 76$. Jean de Maure apparaît également dans ces comptes, pour le versement d'une somme de 139 livres (ibid., p. 191, $\mathrm{n}^{\circ}$ 143). Geoffroy de Guignen a quant à lui été dédommagé pour trois voyages entrepris en Flandre, et l'abbé de Prières, pour l'octroi d'un cheval (dom Morice, Preuves..., t. I, op. cit., col. 1197). Thomas et Geoffroi d'Anast apparaissent dans les comptes de 1303 (LA BORDERIE, Arthur de, "Recueil d'actes... ", op. cit., respectivement p. $188, \mathrm{n}^{\circ} 89$ et p. 189 $\left.n^{\circ} 107\right)$. Enfin, Aubry de Baudement n'est pas étranger non plus au fait des finances : il y a de très fortes probabilités pour que ce soit lui l'auteur des " comptes d'Auberi " publiés par LA BORDERIE, Arthur de, "Recueil d'actes... ", loc. cit., poursuivi dans Bulletin et mémoires de la Société archéologique d'Ille-et-Vilaine, t. xxII, 1893, p. 184-232, n xxv.

89. Tous deux ont ainsi été désignés, avec Raoul le Tort, pour régler un différend opposant Henri d'Avaugour et Alain de Rohan, en 1294 (dom Morice, Preuves..., t. I, op. cit., col. 1116). En 1298, ils ont été chargés, avec Guillaume Hay, de mettre fin au différend entre le duc et Roland de Dinan concernant la vicomté de Léon, en 1298 (ibid., col. 1127).

90. Tous deux avaient été chargés, avec le prieur des Dominicains de Dinan de procéder à la collecte de fonds pour la croisade à laquelle avait appelé en 1300 Boniface VIII (1294-1303) (MARTIN, Hervé, Les ordres mendiants en Bretagne (vers 1230-vers 1530), Paris, Klincksieck, 1975, p. 150).

91. Roland Le Lombard et Jean Le Roy, outre le rôle prépondérant qu'ils sont amenés à jouer dans la liquidation de la succession de Jean II, et accessoirement, des dettes 
Pourquoi en sont-ils venus à s'occuper des dettes de Pierre de Bretagne, alors même qu'il ne s'agit pas de la succession de ce dernier? Plusieurs raisons peuvent être avancées : en premier lieu, les volontés du défunt. Jean II a clairement indiqué, dans son testament, que ses exécuteurs devaient régler pour lui ses dettes et réparer les torts qu'il avait pu causer ${ }^{92}$. Or, il a protégé son fils des plaintes déposées contre lui pour ses déprédations. Il convenait donc d'indemniser ces plaignants, qui réclament à la fois contre Pierre pour son comportement et contre le défunt duc pour sa négligence. Plus largement, le duc a aussi enjoint à ses exécuteurs testamentaires de régler " toutes autres choses queles que eles soient e comant qu'eles soient apelles, soient rendues, restabliz, laissiez et delivrez a ceux qui raison i auront ${ }^{93}$ ". La définition est suffisamment vague pour laisser la plus grande marge de manœuvre possible aux exécuteurs. Il est probable que ces derniers aient jugé plus prudent d'associer le remboursement des dettes de Pierre de Bretagne au règlement global de la succession de Jean II, afin que le scandale éclabousse le moins possible la famille ducale, l'âme du feu duc en particulier. Par ailleurs, Arthur II (1305-1312), désormais duc de Bretagne, hérite des poursuites judiciaires éventuelles qui ont pu être lancées à l'encontre de son père ${ }^{94}$; il est probable qu'il souhaite lui-même se débarrasser au plus vite de cette source d'ennuis. Enfin, l'attitude de Pierre de Bretagne a peut-être concouru au choix de cette solution. En effet, bien que Jean II ne fasse pas mention, dans son testament, de la moindre donation en faveur de son dernier fils ${ }^{95}$, celui-ci devait sans doute pouvoir escompter de rapides rentrées d'argent suite à la disparition paternelle. Pour peu qu'il se soit engagé vis-à-vis de ses créanciers sur des sommes à venir (et il le fait au moins une fois ${ }^{96}$ ), il a bien fallu ensuite tenir la parole donnée, d'autant qu'elle engageait un fils et frère de duc de Bretagne. Bref,

de Pierre de Bretagne, sont tous deux mentionnés dans les comptes ducaux de 1303 (LA Borderie, Arthur de, "Supplément aux actes... ", op. cit., p. 185, n 54 et p. 188, n 88, pour le premier et p. $184, \mathrm{n}^{\circ} 51$ pour le second). Pour Roland Le Lombard, l'on peut même remonter jusqu'à 1288, année lors de laquelle il rend compte de la gestion de la ferme de Quimperlé (LA BorderIE, Arthur de, "Recueil d'actes... ", loc. cit., p. 266, n ${ }^{\circ}$ LXVIII et n ${ }^{\circ} 91$, ainsi que p. $\left.276, \mathrm{n}^{\circ} 178\right)$.

92. "Premièrement, je veil e commant que par les mains de mes aumosniers e executours dessous nommes, mes debtes et mes laiz soient paies, mes amendements faiz, toutes surprinses que je ai faites, toutes choses que je ay mal aquises de terres, de rentes, de deniers" (Arch. dép. de Loire-Atlantique, E 20, ${ }^{\circ}$ 1).

93. Arch. dép. de Loire-Atlantique, E $20, \mathrm{n}^{\circ} 1$.

94. Ce fait est attesté au moins une fois : Guillaume, recteur de Lécousse, et sa mère Jeanne, abandonnent toutes poursuites à l'encontre des exécuteurs testamentaires de Jean II et à l'encontre d'Arthur II, contre 30 livres de monnaie courante (voir annexe 2, pièce $\left.n^{\circ} 10\right)$.

95. Ceci étant, Arthur et Jean, frères aînés de Pierre, ne sont mentionnés que comme futurs gestionnaires de la somme de 30000 livres tournois que le duc prévoit afin d'envoyer des chevaliers et hommes d'armes en Terre sainte, au premier passage qui aura lieu (Arch. dép. de Loire-Atlantique, E 20, n 1). Pierre n'étant cependant absolument pas digne de confiance dès qu'il s'agit d'argent, le duc ne lui confie aucune mission de ce genre.

96. C'est vis-à-vis de Guillaume Le Sergent que Pierre s'engage, le 15 avril 1306, " desus le primer paement que nous devons avoer des exequtors nostre chere signor de pere ou 
pour liquider ses dettes, Pierre a eu la chance d'appartenir à une famille ducale soucieuse de préserver son honneur, mais aussi de voir son père disparaître prématurément, ce qui lui a permis de payer ses dettes dans la foulée. C'est d'ailleurs très probablement à ce décès inopiné que l'on doit la connaissance des tribulations financières de Pierre de Bretagne. Si son père avait vécu, il aurait sans doute veillé à étouffer les exactions de son fils, comme il l'a toujours fait.

Cela ne s'est cependant pas fait sans heurts. Pierre se targuait de pouvoir compter sur le soutien financier des exécuteurs testamentaires de Jean II, ainsi que sur son frère aîné Arthur II; en tout cas, il en fait mention, le 15 avril 1306, lorsqu'il déclare vouloir rembourser Guillaume Le Sergent " desus le primer paement que nous devons avoer des exequtors nostre chere signor de pere ou de la graece que nostre chere frere le duc de Bretaigne nous a gardé ${ }^{97}$ ". Peut-être ce soutien n'allait-il pas sans arrièrepensée, mais le fait est que l'objectif restait le même que sous Jean II : sauvegarder les apparences d'un proche du duc. Néanmoins, Pierre a manqué s'aliéner les soutiens en question.

Peut-être a-t-il dû se montrer assez empressé de profiter de l'occasion, ce qui a indisposé les exécuteurs testamentaires; ou peut-être a-t-il eu des demandes financières telles qu'elles ont été jugées intenables? L'opposition entre les deux parties a été en tout cas suffisamment sérieuse pour nécessiter la conclusion d'une paix entre elles : la date exacte en est inconnue, mais Pierre fait par deux fois allusion à ce fait à l'automne 1306 et les exécuteurs testamentaires le rappellent une fois, en $1308^{98}$.

La conclusion de cette paix prévoit notamment qu'une somme de 6000 livres de faible monnaie serait comptée uniquement pour le règlement des dettes de Pierre de Bretagne. Le 19 octobre 1306, Pierre mentionne explicitement : "La somme de sis mille libres de feble monae que vous avez retenu o vous pour poier pour nous segont la forme de pez que nous avons feite $o$ vous ${ }^{99}$. " À partir de cette date et jusqu'au 10 mars 1308, il est question de cette somme dans dix autres actes ${ }^{100}$; neuf d'entre eux sont émis par Pierre, qui, à chaque fois, s'adresse aux exécuteurs testamentaires de son père en leur demandant de payer tel ou tel montant, à déduire des 6000 livres.

Sur cette importante somme, les exécuteurs en font remettre les deux tiers, soit 4000 livres de faible monnaie, à Pierre lui-même par Robert du

de la graece que nostre chere frere le duc de Bretaigne nous a gardé " (GAONACH, Marie, Jean II..., op. cit., p. 175).

97. GAONACH, Marie, Jean II..., op. cit., p. 175.

98. Le 19 octobre 1306, il évoque, en s'adressant aux exécuteurs testamentaires de son père : "la forme de la pes que nous avons fete o vous " (GAONACH, Marie, Jean II..., op. cit., p. 177) ; il la mentionne également le 19 novembre de la même année, ainsi que le 16 janvier 1307 et le 10 mars 1308 (GAONACH, Marie, ibid., p. 179-180, 182 et 186). Enfin, le 23 mars 1308, les exécuteurs testamentaires reviennent sur « la finaison que nous femes o monsor Pierres de Bretaigne" (ibid., p. 187).

99. GaONACH, Marie, Jean II..., op. cit., p. 178.

100. Respectivement 5 en 1306, 4 en 1307 et le dernier en 1308. 
Change, bourgeois de Nantes (pour 3000 livres), et Roland Le Lombard, de Quimperlé (pour 1000 livres) ${ }^{101}$, et ce probablement pour qu'il règle luimême certaines de ses très nombreuses obligations financières. Ils gardent le reste, que l'on peut donc évaluer à 2000 livres. On pourrait estimer que ces deux sommes étaient en fait distinctes; pour ma part, je pense que les 4000 livres versées à Pierre sont bel et bien issues des 6000 livres décomptées au départ. En effet, avant le versement de 4000 livres, Pierre parle toujours précisément de " la somme de sis mille libres de feble monae que vous avez retenu ${ }^{102}$ ». En revanche, après le versement des 4000 livres, il ne parle désormais que de " la somme que vous nous devez " ou " que vous avez retenue ", sans plus faire allusion au montant originel ${ }^{103}$.

Les exécuteurs ont dû garder le reliquat pour les dédommagements rentrant strictement dans le cadre du règlement de succession (le reste étant pour que Pierre règle discrètement d'autres dettes). Il n'est toutefois pas certain que cela ait suffi. En effet, si l'on fait le compte des sommes déboursées et pour lesquelles il est précisément rappelé qu'elles sont prises sur l'argent bloqué en faveur de Pierre, l'on aboutit à un total de 1298 livres, 8 sous et 8 deniers, ce qui n'excède pas le montant prévu. En revanche, si l'on considère la totalité de ce qu'ils ont versé, que ce soit directement par eux-mêmes ou par d'autres intermédiaires ${ }^{104}$, le total excède visiblement les 2000 livres mises de côté : les seuls paiements en faible monnaie représentent en effet 2128 livres, 12 sous et 6 deniers, auxquels s'ajoutent 524 livres de monnaie courante et 390 livres, 12 sous et 2 deniers de bonne monnaie ${ }^{105}$.

Le remboursement des dettes de Pierre de Bretagne s'est effectué suivant les sessions tenues par les exécuteurs testamentaires, pour le règlement de l'ensemble des sommes dues par le défunt Jean II ${ }^{106}$. L'année 1306 débute modestement avec 4 actes émis de mars à mai; puis 7 sont rédigés aux mois d'octobre et de novembre; 19 sont scellés en 1307, dont 12 au seul mois de janvier. Enfin, 7 derniers actes sont rédigés en 1308 (4 en mars et 3 en mai) ${ }^{107}$.

101. Pierre reconnaît avoir reçu cet argent le 19 octobre 1306 (GAONACH, Marie, Jean II..., op. cit., p. 177).

102. Acte du 19 octobre 1306, phrase qui revient également dans un second acte, émis à la même date (GAONACH, Marie, Jean II..., op. cit., p. 176-178).

103. Expression qui se retrouve à 9 reprises entre le 19 novembre 1306 et le 10 mars 1308 . 104. Les exécuteurs testamentaires procèdent à la quasi-totalité des paiements; Roland Le Lombard assume deux versements et Jean Le Roy, un versement, outre les 4000 livres qu'ils remettent à Pierre de Bretagne.

105. Ainsi, pour être tout à fait exacte, que 20 livres tournois et 137 livres, 6 sous et 8 deniers dont la valeur monétaire n'est pas précisée.

106. Marie GaONACH avait déjà pointé le fait que, au vu des dates d'émissions d'actes, les exécuteurs testamentaires de Jean II s'étaient organisés en sessions pour effectuer leurs dédommagements (Les actes de Jean II..., op. cit., p. 78).

107. Ce qui donne une totalité de 37 actes correspondant aux années 1306-1308, sur les 39 conservés parmi les quittances relatives à la succession de Jean II. Les deux derniers actes datent quant à eux de 1290 et concernent une très ancienne dette de Pierre de Bretagne. 
Les sources sont ici suffisantes pour mettre en évidence le processus de dédommagement ${ }^{108}$ : soit Pierre s'adresse lui-même aux exécuteurs testamentaires, leur demandant de payer telle somme à telle personne; soit le débiteur s'adresse directement aux dits exécuteurs et prouve sa bonne foi, avec des lettres de Pierre à l'appui, ou par témoins ${ }^{109}$. Les exécuteurs effectuent ensuite le paiement du montant requis, contre une quittance de remboursement délivrée par le bénéficiaire.

\section{Religieux, bourgeois ou veuves : les créanciers et plaignants de Pierre de Bretagne}

Le moins que l'on puisse constater est que Pierre de Bretagne fait feu de tout bois. Il a affaire à des profils de personnes très divers, pour différents montants. Il est endetté aussi bien envers des ecclésiastiques séculiers, tel le clerc Richard de Maure, ou réguliers, tel l'abbé de Prières, qu'envers des marchands, des bourgeois ou des veuves. On constate tout de même, sans surprise, dans la petite quarantaine des créanciers ${ }^{110}$, la présence de plusieurs personnes intéressées au maniement des finances ducales, tels Roland Le Lombard, Jean Le Roy ou Bertrand de Chaveignes ${ }^{111}$. On relève aussi les noms de cinq ecclésiastiques; la plupart des autres personnes sont tout simplement des gens à qui il ne paye pas ce qu'il leur achète et qui n'apparaissent qu'à cette occasion. C'est pourquoi il est impossible de détailler davantage qui ils sont ou les raisons pour lesquelles Pierre s'est précisément endetté envers eux ou pour lesquelles il s'en est pris à eux, d'autant que tous n'apparaissent soit qu'une seule fois, soit pour la même affaire ${ }^{112}$.

108. Celui-ci correspond d'ailleurs à ce qu'avait indiqué Jean II dans son testament pour le règlement de ses dettes et autres : «E vueil que mes executeurs aient la connoissance de toutes les choses que l'an lour demandera pour my, en debtes, en torz faiz, en sourprinses, en amendemenz e en totes autres choses, e ainsinc comme il verront que il fera a faire, que il puissent rendre e delivrer o tel enformement e teles preuves que il verront que il devra soffire pour la delivrance e execution doudit testament, e pour le dechargement de m'ame" (Arch. dép. de Loire-Atlantique, E 20, n 1).

109. Ainsi, Jean Dupuis prouve ses dires grâce à des lettres de Pierre de Bretagne (voir annexe 2 , pièce $n^{\circ} 6$ ); en revanche, d'autres font intervenir des témoins, tel Guillaume Le Sergent, invoquant de "bons témoins " (voir annexe 2 , pièce $\mathrm{n}^{\circ} 9$ ).

110. 31 créanciers ont pu être dénombrés (Renaud Gicquel, Pierre Clavereul, Guillaume, recteur de Lécousse et Jeanne, mère de ce dernier, ont été mis à part : ils ne sont pas à proprement parler créanciers de Pierre de Bretagne, ils réclament un dédommagement pour les dommages qu'ils ont subis); cependant, restent à part les boulangers, poissonniers et bouchers de Rennes dont le nombre n'est pas spécifié (GAONACH, Marie, Jean II..., op. cit., p. 185). En les incluant, l'on doit arriver à une petite quarantaine de personnes.

111. L'on a vu plus haut que Roland Le Lombard et Jean Le Roy étaient mentionnés en 1303 dans les comptes ducaux; Bertrand de Chaveignes s'y trouve également, en 1288 (il est alors mentionné comme receveur à Rennes), 1289, 1300 et 1303 (LA BORDERIE, " Recueil d'actes..." "loc. cit., p. 268, n CLXVIII, et n 100, ainsi que p. 277, n 182 et "Supplément aux actes... ", loc. cit., p. 181, n 18 et p. 191, n 139).

112. Ainsi, Richard de Maure est mentionné dans trois actes différents, toujours pour exiger le remboursement de ses 40 livres de monnaie courante; le nom de Guillaume Le Sergent revient deux fois, toujours pour obtenir le dédommagement de son cheval. 
De plus, lorsqu'il est possible de savoir l'origine géographique de ces différents plaignants, il appert que ceux-ci sont assez dispersés : il s'en trouve aussi bien à Paris qu'à Ploërmel, en passant par Rennes, Visseiches ou Quimperlé. Borgne de Douai, Bernard Caone, ou Pierre Le Flament, semblent aussi attester de lointaines créances; j'émets cependant une réserve à leur sujet, dans la mesure où deux d'entre eux sont des marchands. Pierre de Bretagne n'est sans doute pas allé jusqu'à Douai ou Limours-en-Hurepoix pour les rencontrer : il est plus probable que leurs chemins se sont croisés dans la capitale ${ }^{113}$.

Sur l'ensemble des sommes versées par lui-même, ou pour son compte, trois sont très modestes : il s'agit de 60 sous de faible monnaie, et de 40 sous et 36 sous de bonne monnaie, versés à trois veuves et à une dénommée Alix de La Touche. Deux montants tournent autour de 10 livres (respectivement 9 livres de bonne monnaie, 10 livres de faible monnaie et 11 livres de monnaie courante). Pour le reste, trois des sommes se situent entre 20 et 50 livres, sept sont comprises dans une fourchette entre 50 et 100 livres et huit, tout de même, sont supérieures à 100 livres, avec un record de 770 livres de monnaie courante, dues à Bernard Caone, marchand de Limours-en-Hurepoix. Ces huit plus fortes sommes sont dues, pour quatre d'entre elles, à des marchands; deux sont dues à des bourgeois; une à un curé; et la dernière à Pierre Le Flament, dont, pour n'en avoir pas trouvé trace, j'ignore ce qu'il fait par ailleurs.

Ces sommes sont réparties en trois grandes catégories : d'une part, les emprunts; d'autre part, les achats à crédit; enfin, les dédommagements dus pour les déprédations commises par Pierre. Les achats semblent évidents pour les marchands de chevaux, les boulangers, bouchers et poissonniers. Il est en revanche plus vraisemblable que l'argent dû à certains des exécuteurs testamentaires du duc Jean II ou à des bourgeois de Ploërmel et de Rennes sont le fruit d'emprunts. Le terme "dette " se retrouve cinq fois ${ }^{114}$, et même lorsqu'il est employé, il n'est pas nécessairement significatif, car il désigne toute somme d'argent due, quelle qu'en soit la cause. Pierre de Bretagne n'est pas plus explicite lorsqu'il reçoit 20 livres tournois pour l'abbé de Prières " au cui ge les devoie ", dit-il ${ }^{115}$; vu le débiteur en question, l'on se doute qu'il s'agit là d'un emprunt, mais cela n'est pas dit. Seuls deux actes mentionnent explicitement un prêt : il s'agit des 20 livres prêtées par Richard de Maure en 1290 et des 40 sous de bonne monnaie dus à Peronne, veuve de Guillaume Morou, pour le prêt fait par son époux ${ }^{116}$.

113. Borgne de Douai est l'un des trois marchands de chevaux auprès desquels Pierre est endetté en 1291 et qui cause son emprisonnement à Paris (c'est ce lieu de détention qui me porte à croire que Paris était le lieu de rencontre de Pierre et de ce marchand); Bernard Caone est un marchand originaire de Limours-en-Hurepoix.

114. L'un d'entre eux vaut pour quatre personnes, ce qui fait un total de neuf dettes.

115. GAONACH, Marie, Jean II..., op. cit., p. 180.

116. Il est question de " deners prestez " (GAONACH, Marie, Jean II..., op. cit., p. 173). 
Concernant les emprunts, les plus élevés d'entre eux ont été contractés, sans surprise, auprès de personnes susceptibles de pouvoir rapidement mettre à disposition le montant requis. Il s'agit d'un ecclésiastique, l'abbé de Prières, qui prête jusqu'à 131 livres ${ }^{117}$; de deux bourgeois, à savoir Jean Le Roy, bourgeois de Ploërmel, auquel sont dues 193 livres et 9 sous de faible monnaie, et Pierre Paquier, bourgeois de Rennes, pour 102 livres, 6 sous et 8 deniers de bonne monnaie; enfin, 207 livres était à rendre à Pierre Le Flament, personnage malheureusement inconnu par ailleurs ${ }^{118}$.

Quant à ses déprédations diverses et variées, elles concernent 11 plaignants (défunts compris), soit plus d'un quart des bénéficiaires de versements financiers de la part des exécuteurs testamentaires; et parmi ces plaignants, trois sont ecclésiastiques. Aucune raison n'est cependant donnée quant à la cause expliquant les méfaits commis. Prendre du vin à Jean Dupuis peut s'expliquer par une occasion de boire à peu de frais ${ }^{119}$. En revanche, qu'ont fait Eudes Frelon et son père pour que leurs bois et vignes soient ravagés? Qu'ont fait Guillaume, recteur de Lécousse, et sa mère Jeanne, qui justifierait le pillage de leur maison de Saint-Aubin-duCormier? Pierre avait-il une raison précise? Ou bien était-il tout simplement en quête d' " amusement " aux dépens d'autrui?

Quoi qu'il en soit, les exécuteurs testamentaires parviennent à éteindre toute action en justice grâce à de multiples dédommagements, excepté pour deux des plaignants : Guillaume, recteur de Lécousse, et sa mère, victimes de plusieurs pillages et visiblement molestés par les gens de Pierre de Bretagne, acceptent de cesser toute poursuite à l'encontre des exécuteurs testamentaires de Jean II et à l'encontre d'Arthur II; en revanche, ils maintiennent leurs plaintes contre Pierre de Bretagne, ses serviteurs et toute personne responsable de ce qui leur a été infligé ${ }^{120}$.

La succession de son père est d'autant plus instructive pour les historiens qu'elle aura permis de mieux cerner le personnage. Sans cela, nous ne l'aurions vu que sous l'angle du chevalier, cadet de famille noble, effectuant son service auprès du roi de France, comme le prouve sa participation aux campagnes de Flandre de 1302 et $1303^{121}$. Un chevalier parmi d'autres, en somme.

117. L'abbé de Prières est le seul à avoir prêté plusieurs fois de l'argent à Pierre, dans la limite des sources subsistantes (il est probable que Roland Le Lombard, pour ne citer que lui, a dû prêter de l'argent plus d'une fois, mais rien ne le prouve) : 20 livres tournois, puis 131 livres de faible monnaie et enfin, 87 livres, 6 sous et 8 deniers lui sont remboursés en 1306 (voir le tableau récapitulatif des créanciers et des plaignants).

118. Seuls les prêts supérieurs à 100 livres ont été pris en compte, quelle que soit la valeur de la monnaie (voir le tableau récapitulatif des créanciers et des plaignants).

119. D'autant que Pierre lui avait laissé des lettres, sans doute une reconnaissance de dette pour le préjudice commis (voir annexe 2 , pièce $n^{\circ} 6$ ).

120. Voir annexe 2 , pièce $n^{\circ} 10$.

121. Pierre prend part à la bataille de Courtrai (voir l'itinéraire en annexe 1). 
Tableau récapitulatif des créanciers et plaignants, ainsi que des sommes versées par, ou pour le compte de Pierre de Bretagne à l'occasion du règlement de la succession de Jean II, établi d'après les mandements de payer et les quittances (Source : corpus de documents. Les années indiquées sont celles du règlement effectif des dettes.)

\begin{tabular}{|c|c|c|c|c|}
\hline Année & Nom du créancier & Statut ou profession & $\begin{array}{c}\text { Objet de la récla- } \\
\text { mation }\end{array}$ & Somme versée \\
\hline 1306 & Abbé de Prières & $\begin{array}{l}\text { Religieux régulier et } \\
\text { exécuteur testamen- } \\
\text { taire de Jean II }\end{array}$ & Dette & 20 livres tournois \\
\hline 1306 & Bernard Caone & $\begin{array}{l}\text { Marchand de Limours- } \\
\text { en-Hurepoix }\end{array}$ & Dette & $\begin{array}{l}370 \text { livres de monnaie } \\
\text { courante versées (sur } \\
770 \text { livres de dettes) }\end{array}$ \\
\hline 1306 & Jean de Beaumont & $\begin{array}{l}\text { Clerc de Pierre de } \\
\text { Bretagne }\end{array}$ & Dette & $\begin{array}{l}73 \text { livres de monnaie } \\
\text { courante }\end{array}$ \\
\hline 1306 & Pierre des Fourne & & Dette & 50 livres \\
\hline 1306 & Pierre le Flament & & Dette & $\begin{array}{l}207 \text { livres de faible } \\
\text { monnaie }\end{array}$ \\
\hline 1306 & Guillaume de Guignen & $\begin{array}{l}\text { Exécuteur testamen- } \\
\text { taire de Jean II }\end{array}$ & Dette & $\begin{array}{l}70 \text { livres de faible } \\
\text { monnaie }\end{array}$ \\
\hline 1306 & Nicolas Chrétien & & Dette & $\begin{array}{l}20 \text { livres de faible } \\
\text { monnaie }\end{array}$ \\
\hline 1306 & Femme Colet & & Dette & $\begin{array}{l}10 \text { livres de faible } \\
\text { monnaie }\end{array}$ \\
\hline 1306 & Abbé de Prières & $\begin{array}{l}\text { Religieux régulier et } \\
\text { exécuteur testamen- } \\
\text { taire de Jean II }\end{array}$ & Dette & $\begin{array}{l}131 \text { livres de faible } \\
\text { monnaie }\end{array}$ \\
\hline 1306 & Roland Le Lombard & De Quimperlé & Dette & $\begin{array}{l}75 \text { livres, } 9 \text { sous et } \\
6 \text { deniers de bonne } \\
\text { monnaie }\end{array}$ \\
\hline 1306 & Abbé de Prières & $\begin{array}{l}\text { Religieux régulier et } \\
\text { exécuteur testamen- } \\
\text { taire de Jean II }\end{array}$ & Dette & $\begin{array}{l}87 \text { livres, } 6 \text { sous et } 8 \\
\text { deniers }\end{array}$ \\
\hline 1306 & Jean Le Roy & Bourgeois de Ploërmel & Dette & $\begin{array}{l}193 \text { livres, } 9 \text { sous de } \\
\text { faible monnaie }\end{array}$ \\
\hline 1306 & $\begin{array}{l}\text { Guillaume Pignel et sa } \\
\text { sœur Jeanne }\end{array}$ & & Dette & $\begin{array}{l}68 \text { livres et } 10 \text { sous de } \\
\text { faible monnaie }\end{array}$ \\
\hline 1306 & $\begin{array}{l}\text { Guillaume Quintela- } \\
\text { men }\end{array}$ & & Dette & $\begin{array}{l}57 \text { livres, } 13 \text { sous, } \\
6 \text { deniers de faible } \\
\text { monnaie }\end{array}$ \\
\hline 1307 & $\begin{array}{l}\text { Guillaume de La Bou- } \\
\text { teillerie et son épouse } \\
\text { Perrone }\end{array}$ & & $\begin{array}{l}\text { Quatre pipes de vin } \\
\text { d'Anjou et un tonneau } \\
\text { de vin de Gascogne } \\
\text { (ainsi qu'un cheval, } \\
\text { deux vaches et une } \\
\text { maison qu'avait récu- } \\
\text { pérés Jean II) }\end{array}$ & $\begin{array}{l}40 \text { livres de bonne } \\
\text { monnaie }\end{array}$ \\
\hline 1307 & Richard de Maure & Clerc & $\begin{array}{l}20 \text { livres de prêt et } \\
20 \text { livres pour l'achat } \\
\text { d'un cheval }\end{array}$ & $\begin{array}{l}40 \text { livres de monnaie } \\
\text { courante }\end{array}$ \\
\hline
\end{tabular}




\begin{tabular}{|c|c|c|c|c|}
\hline 1307 & Jean Vacher & $\begin{array}{l}\text { Prêtre recteur de } \\
\text { Groys }\end{array}$ & $\begin{array}{l}\text { Dettes et dommages } \\
\text { causés par Pierre et } \\
\text { ses gens }\end{array}$ & $\begin{array}{l}72 \text { livres, } 14 \text { sous de } \\
\text { bonne monnaie }\end{array}$ \\
\hline 1307 & Pierre Paquier & Bourgeois de Rennes & Dette & $\begin{array}{l}102 \text { livres, } 6 \text { sous et } \\
8 \text { deniers de bonne } \\
\text { monnaie }\end{array}$ \\
\hline 1307 & $\begin{array}{l}\text { Guillaume de Vezin et } \\
\text { Jean Gaepin }\end{array}$ & Selliers de Rennes & Dette & $\begin{array}{l}320 \text { livres de faible } \\
\text { monnaie }\end{array}$ \\
\hline 1307 & Mahaut et Peronne & Veuves & $\begin{array}{l}\text { Achat à crédit de pain } \\
\text { de froment }\end{array}$ & $\begin{array}{l}60 \text { sous de faible } \\
\text { monnaie }\end{array}$ \\
\hline 1307 & Perronne & Veuve & $\begin{array}{l}\text { Emprunt à feu son } \\
\text { époux }\end{array}$ & $\begin{array}{l}40 \text { sous de bonne } \\
\text { monnaie }\end{array}$ \\
\hline 1307 & Alix de la Touche & & Dette & $\begin{array}{l}36 \text { sous de bonne } \\
\text { monnaie }\end{array}$ \\
\hline 1307 & $\begin{array}{l}\text { Pierre Raoul de } \\
\text { Penhars }\end{array}$ & & Deux bœufs & $\begin{array}{l}10 \text { livres de faible } \\
\text { monnaie }\end{array}$ \\
\hline 1307 & Jean Orquant & & Dette & $\begin{array}{l}9 \text { livres de bonne } \\
\text { monnaie }\end{array}$ \\
\hline 1307 & $\begin{array}{l}\text { Renaud Gicquel et } \\
\text { Pierre Clavereul }\end{array}$ & $\begin{array}{l}\text { Renaud est dit habi- } \\
\text { tant de Rennes; Pierre } \\
\text { est son beau-fils }\end{array}$ & $\begin{array}{l}\text { Prises aux dépens } \\
\text { de leurs biens, par } \\
\text { Pierre de Bretagne et } \\
\text { ses gens }\end{array}$ & $\begin{array}{l}126 \text { livres et } 7 \text { sous de } \\
\text { faible monnaie }\end{array}$ \\
\hline 1307 & & $\begin{array}{l}\text { Panetiers de Rennes } \\
\text { ou des environs }\end{array}$ & Achats à crédit & $\begin{array}{l}400 \text { livres de faible } \\
\text { monnaie }\end{array}$ \\
\hline 1307 & & $\begin{array}{l}\text { Bouchers et poisson- } \\
\text { niers de Rennes }\end{array}$ & Achats à crédit & $\begin{array}{l}200 \text { livres de faible } \\
\text { monnaie }\end{array}$ \\
\hline 1307 & Jean Dupuis & & $\begin{array}{l}\text { Prise de vin par Pierre } \\
\text { de Bretagne et ses } \\
\text { gens }\end{array}$ & $\begin{array}{l}12 \text { livres de faible } \\
\text { monnaie }\end{array}$ \\
\hline 1307 & Eudes Frelon & $\begin{array}{l}\text { Originaire du diocèse } \\
\text { de Rennes }\end{array}$ & $\begin{array}{l}\text { Ravages causés à ses } \\
\text { bois et vignes et à } \\
\text { ceux de son père par } \\
\text { Pierre de Bretagne et } \\
\text { ses gens }\end{array}$ & $\begin{array}{l}100 \text { livres de bonne } \\
\text { monnaie }\end{array}$ \\
\hline 1307 & $\begin{array}{l}\text { Betrand de Cha- } \\
\text { veignes }\end{array}$ & Curé de Visseiche & Dette & $\begin{array}{l}300 \text { livres de faible } \\
\text { monnaie }\end{array}$ \\
\hline 1308 & Geoffroy Choan & Clerc & Dette & $\begin{array}{l}60 \text { livres de bonne } \\
\text { monnaie courante }\end{array}$ \\
\hline 1308 & Guillaume Le Sergent & & Prise d'un cheval & $\begin{array}{l}11 \text { livres de monnaie } \\
\text { courante }\end{array}$ \\
\hline 1308 & Guillaume et Jeanne & $\begin{array}{l}\text { Prêtre recteur de } \\
\text { Lécousse et sa mère }\end{array}$ & $\begin{array}{l}\text { Pillage de leurs biens, } \\
\text { notamment d'une } \\
\text { maison à Saint-Aubin- } \\
\text { du-Cormier }\end{array}$ & $\begin{array}{l}30 \text { livres de monnaie } \\
\text { courante }\end{array}$ \\
\hline
\end{tabular}

Pierre de Bretagne sort pourtant de l'ordinaire. C'est même un personnage détonnant dans la famille ducale de la maison de Dreux, dont aucun membre ne s'est ainsi distingué. Pierre de Dreux (1213-1237) a pu être un vassal turbulent ou Jean $\mathrm{I}^{\mathrm{er}}$, un seigneur retors, mais aucun n'a été à la fois dévoyé et dépensier tout à la fois. De même, il n'y a pas d'individu semblable parmi les cadets de cette maison; la plupart d'entre eux servent plus 
ou moins les intérêts du duc régnant, tel Pierre de Bretagne (1241-1268), fils du duc Jean $\mathrm{I}^{\mathrm{er}}$ ou Jean, comte de Richemont (1266-1334). Guy de Bretagne (1287-1331) fait exception, cherchant plutôt à se constituer une seigneurie indépendante de son frère aîné Jean III (1312-1341); au moins avait-il de l'ambition, contrairement à Pierre de Bretagne, qui n'avait que l'envergure d'un délinquant local ${ }^{122}$.

L'on ignore ce qu'il devient par la suite ${ }^{123}$. Il ne semble pas qu'Arthur II ait eu pour son cadet la même indulgence que Jean II. En tout cas, il n'y a nulle trace d'aucune dépense, ni d'un quelconque versement en sa faveur après le 3 mai 1308, mais cela n'est certainement pas dû à un soudain esprit d'économie de sa part; il serait en effet douteux qu'un tel prodigue ait changé du jour au lendemain. La vie de ses parents, frères et sœurs ne renseigne pas davantage, car il ne semble pas avoir été très proche de sa famille, ni avoir été très recherché par elle (ce qui peut se comprendre, vu son attitude, et surtout vu ce qu'il coûtait). Il n'apparaît même dans aucune source entre 1308 et mars 1314. Il est apparemment encore vivant cette année-là, étant mentionné de manière très formelle dans l'acte par lequel le roi Philippe IV valide le don de la vicomté de Limoges à Guy de Bretagne par son frère aîné Jean III ${ }^{124}$. Il disparaît définitivement par la suite. Le silence imposé sur cette honte de la famille laisse dans l'ombre aussi bien son existence que la date de son décès, tout fils, frère, puis oncle de duc qu'il était.

122. Sur Pierre de Bretagne, fils du duc Jean $\mathrm{I}^{\mathrm{er}}$, je me permets de renvoyer à ma propre étude : "Jean Ir , duc de Bretagne, comte de Richemont et Pierre de Bretagne, chevalier, son fils " et sur Guy de Bretagne, à mon intervention sur " Deux titulaires pour une vicomté? Les démêlés d'Isabelle d'Espagne, duchesse de Bretagne, vicomtesse de Limoges, avec Guy de Bretagne, vicomte de Limoges ", in La vicomté de Limoges sous les ducs de Bretagne, journée d'études, Limoges, 30 janvier 2015. Pour Jean, comte de Richemont (1306-1334), fils de Jean II et donc frère aîné de Pierre de Bretagne, voir LuBimenKo, Inna, Jean de Bretagne, comte de Richmond (1266-1334), Lille, le Bigot frères, 1908, p. 5-6.

123. Il a été plusieurs fois écrit qu'il serait décédé en juillet 1312, des suites d'un coup de pied donné par un cheval (dom LoBineau, Histoire de Bretagne..., t. I, op. cit., p. 296, repris par dom Morice, Histoire de Bretagne..., t. I, op. cit., p. 229; LA BORDERIE, Arthur de, Histoire de Bretagne, t. III, De l'an 995 après J.-C. à l'an 1364, Rennes, J. Plihon et L. Hommay, 1898, éd. fac-similé, Mayenne, J. Floch, 1972, p. 401). Toutefois, ce n'est pas lui qui est la victime : il s'agit de son neveu et homonyme Pierre de Bretagne, fils d'Arthur II (12891312), le troisième Pierre de Bretagne qu'a compté la maison ducale de Dreux en un peu plus d'un siècle.

124. C'est une indication très brève. Guy, bénéficiaire de la vicomté de Limoges, renonce à toute succession qui aurait pu lui advenir dans le futur, suite au décès de ses demifrères et sœurs, nés de son père et de la seconde épouse de celui-ci, Yolande de Dreux; ou suite au décès de Jean de Bretagne, comte de Richemont, ou de Pierre de Bretagne, ses oncles, "ipse Guido renunciavit expresse totali successioni que ipsi posset descendere in futurum, ex morte fratrum suorum et sororum ex Yolendi, quondam ducissa Britannie, noverca eorum procreatorum ac Johanni de Britannia, comitis de Diviti Monte, et etiam Petri de Britannia, fratris ejus militi, avunculorum suorum " (Arch. nat., JJ 49, $\mathrm{f}^{\circ} 97 \mathrm{v}^{\circ}$ ). Jean et Pierre de Bretagne sont alors toujours en vie; dans le cas contraire, le fait qu'ils soient décédés aurait été indiqué. 


\section{Annexe 1 - Itinéraire de Pierre de Bretagne}

Cet itinéraire a été établi d'après le corpus de documents étudié, avec l'appui de certaines archives supplémentaires.

$\begin{array}{lll}\mathbf{1 2 9 1} & \text { 29-30 mai } & \text { Paris } \\ \mathbf{1 2 9 3} & \text { octobre } & \text { Paris }{ }^{125} \\ \mathbf{1 2 9 4} & \text { février } & \text { Paris } \\ \mathbf{1 3 0 2} & \text { 11 juillet } & \text { Courtrai }{ }^{126} \\ \mathbf{1 3 0 3} & \text { août } & \text { au camp d'Arras }^{127} \\ \mathbf{1 3 0 6} & \begin{array}{l}\text { 20 mai } \\ \text { s. d. }\end{array} & \text { Suscinio } \\ & \text { s. d. } & \text { Penmur } \\ & \text { 19 novembre } & \text { Quimperlé } \\ & \text { Ploërmel } \\ \mathbf{1 3 0 7} & \text { 16 janvier } & \text { Ploërmel } \\ & \text { 19 mars } & \text { Quimperlé } \\ & 7 \text { novembre } & \text { Quimperlé }\end{array}$

Annexe 2 - Pièces justificatives

$$
-1 \text { - }
$$

1306, 14 avril.

Pierre de Bretagne reconnaît avoir reçu des exécuteurs testamentaires de son père 130 livres de monnaie courante, ainsi que 370 livres de la dite monnaie, versées au marchand Bernard Caone.

A. Original, parchemin, larg. 175/165 mm x haut. 75/55 mm (haut. de queue, $20 \mathrm{~mm}$ ), scellé sur simple queue de parchemin du sceau de Pierre de Bretagne en cire brune ${ }^{129}$, Arch. dép. de Loire-Atlantique, E 21, $\mathrm{n}^{\circ} 2$.

IndiQUe : GaONACH, Marie, Jean II..., op. cit., p. 199.

125. Pierre est présent à l'occasion de l'émancipation paternelle. Il est bien précisé, dans l'acte, que le principal intéressé est "presentem et expresse consentientem " ou présent et expressément consentant (Arch. dép. de Loire-Atlantique, E 1, n² 2).

126. De même que son frère Jean, vicomte de Limoges, il a été convoqué par lettres du 7 mai 1302 " pour se trouver au camp d'Arras le premier jour de juin prochain, avec armes et chevaux, le mieux qu'il leur seroit possible ", Bibliothèque nationale de France, nouv. acq. fr. $7366, f^{\circ} 24 \mathrm{v}^{\circ}$. Pierre prend ensuite part à la bataille de Courtrai (dom LOBINEAU, Histoire de Bretagne... op. cit., t. I, p. 289).

127. Convoqué au camp d'Arras par lettres du 21 août 1303, Pierre s'est présenté à Arras, ainsi que 25 autres seigneurs bretons (Bibliothèque nationale de France, nouv. acq. fr. $\left.7366, \mathrm{f}^{\circ} 321 \mathrm{v}^{\circ}\right)$.

128. Le jour de l'acte n'est pas spécifié, toutefois, vu que Pierre se trouve à Ploërmel à la fin de l'année 1306 et encore au tout début de l'année 1307, il est très probable qu'il y est resté durant l'hiver. Aussi ai-je situé son passage à Penmur et Quimperlé avant.

129. Résumé d'une main du XIXe siècle : "1306. Reçu de 130 livres payées à Pierre de Bretagne pour fin de compte de 500 livres ». 
Nos, Petrus, filius quondam ducis Britannie, comes Rychemondie, habuimus et recepimus ab exequtoribus testamenti inclite recordacionis domini nostri ducis predicti, sex viginti et decem libras monete currentis per manum Jameti Bertrun, clerici nostri, velut trescentas et sexaginta decem libras ejusdem monete quas dicti exequtores solverunt pro nobis Bernardo Cahone, mercatori de Lymous. Et ita nos tenemur pro pagato de quingentis libris nobis debitis, de gratia karissimi genitoris nostri predicti, ad festum Omnium Sanctorum ultimo preteritum et de omni tempore retroacto. Datum die veneris post Quasimodo, anno Domini $\mathrm{M}^{\circ} \mathrm{CCC}^{\circ}$ sexto.

Nous, Pierre, fils de feu le duc de Bretagne, comte de Richemont, avons eu et reçu des exécuteurs du testament de notre susdit seigneur duc d'illustre souvenir, 130 livres de monnaie courante par la main de Jamet Bertrand, notre clerc, comme 370 livres de cette même monnaie que les dits exécuteurs ont versé pour nous à Bernard Caone, marchand de Limours. Et nous nous sommes ainsi tenu pour payé des 500 livres à nous dues, par la grâce de notre très cher géniteur susdit, à la fête de Toussaint dernièrement passée et de tout temps passé. Donné le vendredi après Quasimodo, en l'an du Seigneur mille trois cent six.

1307 (n. st), 12 janvier.

L'archidiacre de Porhoët fait savoir que Jean Vacher, prêtre recteur de l'église de Groys, pour les dettes que lui devait Pierre de Bretagne, ainsi que pour les dommages occassionnés par lui et ses gens au dit Jean, a reçu des exécuteurs testamentaires de Jean II, 72 livres, 14 sous de bonne monnaie.

A. Original, parchemin, scellé sur simple queue de parchemin du sceau de la cour de l'archidiacre de Porhoët en cire verte ${ }^{130}$, Arch. dép. de Loire-Atlantique, E $22, n^{\circ} 91$.

INDIQUE : GAONACH, Marie, Jean II..., op. cit., p. 206.

Universis presentes litteras inspecturis et audituris, archidiaconus de Porhoet in ecclesia Macloviensis, salutem in Domino. Noverint universi quod coram nobis personaliter constitutus, Johannes Vachier, rector ecclesie de Croys, confessus fuit se amicabiliter composuisse et finasse cum exequtoribus testamenti inclite recordacionis Johannis, ducis Britannie, ultimo deffuncti, de omnibus debitis que eidem Johanni debebat, per litteras et sine litteris, nobilis vir dominus Petrus de Britannia, miles, filius quondam dicti ducis, et de omnibus capcionibus, invasionibus et occupacionibus factis per dictum dominem Petrum, et suos complices et servientes et alios quoscumque racione dicti Petri, de bonis dicti Johannis et de omnibus aliis, in quibus dictus Petrus et sui servientes et gentes poterant obligari quecomque de terra eidem ${ }^{131}$; et pro premissis habuit ab exequtoribus predictis, per manum Rollandi Le Lambart, sexaginta duodecim libras et quatuordecim solidos de bona moneta et de premissis se tenuit pro pagato ab exequtoribus predictis et ipsos quitavit de omnibus a tempore retroacto, et premissa et singula juravit dictus Johannes tenere et contra non venire et ad hoc fuit autem nostra sentencia condampnatus. Datum teste sigillo nostro curie nostre, die jovis post Epiphaniam Domini, anno ejusdem $\mathrm{M}^{\circ} \mathrm{CCC}^{\mathrm{mo}}$ sexto.

130. Mention dorsale du XIXe siècle : « 1307. Reçu de 72 livres payées à Jean Vachier, curé de l'église de Groys pour dégâts commis sur ses terres. "

131. " eidem ", écrit au-dessus de la ligne, $A$. 
À tous ceux qui verront et entendront les présentes lettres, l'archidiacre de Porhoet en l'église de Saint-Malo, salut dans le Seigneur. Sachez que devant nous personnellement comparu, Jean Vacher, recteur de l'église de Groys, a confessé avoir amicalement composé et m'être arrangé avec les exécuteurs du testament de Jean d'illustre souvenir, duc de Bretagne, récemment défunt, de toutes les dettes qu'au même Jean devait, par lettres et sans lettres, le noble homme, le seigneur Pierre de Bretagne, chevalier, fils du feu dit duc, et de tous les prises, ravages et occupations faits par le dit seigneur Pierre, ses complices, serviteurs et qui que ce soit d'autres en raison du dit Pierre, sur les biens du dit Jean et sur tous les autres sur lesquels le dit Pierre, ses serviteurs et gens pouvaient se rendre responsables de quoi que ce soit envers le même, sur sa terre; et pour ce qui est susdit, il a eu des exécuteurs susdits, par la main de Roland Le Lombard, 72 livres et 14 sous de bonne monnaie, il se tint pour payé par les susdits exécuteurs de ce qui est susdit, les a tenu quitte de tout depuis le temps passé, et le dit Jean a juré de tenir ce qui est susdit et chaque point et de ne pas venir encontre, et il a été condamné à cela par notre sentence. Donné, témoin le sceau de notre cour, le jeudi après l'Epiphanie du Seigneur, en l'an du même mille deux cent six.

$$
\text { - } 3 \text { - }
$$

1307 (n. st), 16 janvier. - Ploërmel.

G., archidiacre de Porhoet, atteste que Richard de Maure, clerc, a bien reçu 40 livres des exécuteurs testamentaires du duc Jean II, en dédommagement d'un prêt et de l'achat d'un cheval, dus par Pierre de Bretagne.

A. Original, parchemin, larg. 230/235 $\mathrm{mm}$ x haut. $80 / 65 \mathrm{~mm}$ (haut. de queues, 7 et $8 \mathrm{~mm}$ ), jadis scellé sur deux simples queues de parchemin du sceau de la cour de Porhoët et du sceau de Richard de Maure, tous deux en cire verte ${ }^{132}$, Arch. dép. de Loire-Atlantique, E $22, \mathrm{n}^{\circ} 55$.

INDIQUE : GAONACH, Marie, Jean II, op. cit., p. 202.

Universis presentes litteras inspecturis, G., archidiaconus de Porhoet in ecclesia Macloviensis, salutem in Domino. Noveritis quod coram nobis in jure personaliter constitutus, magister Richardus de Maurra, clericus, confessus fuit se habuisse et recepisse ab exequtoribus testamenti inclite recordationis Johannis, quondam ducis Britannie, nuper defuncti, quadraginta libras de bona moneta, in quibus nobilis vir, dominus Petrus de Britania, dicto magistro tam ex causa mutui quam ex vendicione cujusdem ${ }^{133}$ equi, diu est, tenebatur, prout in litteris dicti domini Petri vidimus continenta dictusque magister dictum nobilem dominum Petrum exequtoresque predictores, sicut predictum, soluit penitus et quitavit, sigillo nostre curie ad petitum dicti magistri una cum sigillo ipsius presentis apposito, in testimonium ${ }^{134}$ premissorum. Datum die lune postea festum beati Hyllarii, anno Domini $\mathrm{M}^{\circ} \mathrm{CCC}^{\circ}$ sexto.

À tous ceux qui verront les présentes lettres, $G$., archidiacre de Porhoet en l'église de Saint-Malo, salut dans le seigneur. Sachez que, ayant comparu en droit devant nous, maître Richard de Maure, clerc, a confessé avoir eu et reçu des exécuteurs du testament de Jean d'illustre souvenir, feu duc de Bretagne, récemment décédé, 40 livres de bonne monnaie, auxquelles le noble homme, le seigneur Pierre de Bretagne, était tenu envers

132. Mention dorsale du XIX ${ }^{\mathrm{e}}$ siècle : «1307, 16 janvier. Reçu de 40 livres payées à Richard de Maure".

133. " cujusdem ", sic, A, pour " cujusdam ".

134. Suivi de "veri ", barré, $A$. 
le dit maître, tant à cause d'un prêt que de la vente d'un cheval, il y a longtemps ${ }^{135}$, ainsi que nous l'avons vu contenu dans les lettres du dit seigneur Pierre et le dit maître a entièrement délié et tenu quitte, comme susdit, le dit noble seigneur Pierre et les susdits exécuteurs, avec apposition aux présentes du sceau de notre cour, à la demande du dit maître, en même temps que de son sceau, en témoignage de ce qui est susdit. Donné le lundi après la fête de saint Hilaire, en l'an du Seigneur mille trois cent six.

1307 (n. st.), 24 janvier

Pierre Raoul de Penhars reconnaît avoir reçu 10 livres de faible monnaie et en tient quitte le défunt duc de Bretagne et ses successeurs.

A. Original, parchemin, larg. 195/170 mm x haut. 70/75 mm (haut. de queue, $9 \mathrm{~mm}$ ), scellé sur simple queue en parchemin du sceau de l'official de Rennes en cire brune ${ }^{136}$, Arch. dép. de Loire-Atlantique, E 22, nº 68.

INDIQUE : GAONACH, Marie, Jean II..., op. cit., p. 202.

Coram nobis in jure personaliter constitutus, Petrus Radulphi de Penherz recognovit se habuisse et recepisse decem libras in debili moneta ab exequtoribus testamenti illustris viri domini Johannis, nuper ducis Britannie et comitis Richemondie, nomine exequtoria predictorum, quitans et liberans dictum dominum Johannem, animam suam, heredes et successores suos quoscumque, ab omnibus forefactis et de omnibus debitis eidem competentis quibuscumque, contra dictum dominum Johannem, quocumque nomine et causa. Datum teste sigillo curie nostre, die martis postea festum beati Vicencii, anno Domini $\mathrm{M}^{\circ} \mathrm{CCC}^{\mathrm{mo}}$ sexto.

Devant nous personnellement comparu en droit, Pierre Raoul de Penhars a reconnu avoir eu et reçu 10 livres de faible monnaie des exécuteurs du testament du brillant homme le seigneur Jean, récemment duc de Bretagne et comte de Richemont, au nom de l'exécution des susdits, tenant quitte et libérant le dit seigneur Jean, son âme, ses héritiers et successeurs quels qu'ils soient, de tous les forfaits et de toutes les dettes lui revenant, quelles qu'elles soient, contre le dit seigneur Jean, en quelque nom et cause que ce soit. Donné témoin le sceau de notre cour, le mardi après la fête de saint Vincent, en l'an du Seigneur mille trois cent six.

$-5-$

1307 (n. st.), 13 mars

Renaud Gicquel et Pierre Clavereul reconnaissent avoir reçu des exécuteurs testamentaires du défunt Jean II 126 livres et 7 sous de faible monnaie, en dédommagement de ce que Pierre de Bretagne leur avait pris.

135. Il y a dix-sept ans, la première reconnaissance de dette de Pierre au sujet de ce cheval date de 1290 .

136. Mention hors teneur, sur le coin supérieur droit de l'acte : "Pro domino Petro de Britannia pro duobus bobus" (pour le seigneur Pierre de Bretagne, pour deux bœufs). Mention dorsale : " 24 janvier 1307. Quittance de 10 livres payées à Pierre Raoul de Penhers ". 
A. Original, parchemin, largeur $160 / 165 \mathrm{~mm} \times$ haut. $55 / 40 \mathrm{~mm}$ (haut. de queues $9 \mathrm{~mm}$ et $6 \mathrm{~mm}$ ), scellé sur deux simples queues de parchemin des sceaux de la cour de Rennes et de Renaud de Clavereul, tous deux en cire brune ${ }^{137}$, Arch. dép. de Loire-Atlantique, E 22, n 48.

INDIQUE : GAONACH, Marie, Jean II..., op. cit., p. $203^{138}$.

Noverint universi quod coram nobis, officialis Redonensis, in jure personaliter constituti, Raginaldus Giquel, civis Redonensis, tam nomine suo quam nomine Petronille, quondam Johannis Leclaveroul et modo dicti Raginaldi uxoris, necnon Petrus dictus Claveroul, filius dictorum Johannis et Petronille, tam nomine suo quam Johannis et Johannis, liberorum dictorum Johannis et Petronille, confessi sunt se habuisse et recepisse ab executoribus inclite recordationis domini defuncti Johannis, nuper ducis Britannie et comitis Richemondie, sex viginti sex libras et septem solidos debilis monete, pro eo quod nobilis vir dominus Petrus de Britannia, miles, filius dicti domini ducis capi fecerat et habuerat de numeratis dictorum Johannis et Raginaldi, contra voluntatem eorumdem, et de quibus clamore detulerant dicto domino duci, tempore quo vivebat, et de qua pecunie summa ${ }^{139}$ fiebat mentio in quodam rotulo tradito eisdem executoribus ex parte dicti domini Petri et quod captio facta fuerat duodecim annis elapsis vel circa, probatione sufficienti super his facta coram predictis executoribus, prout nobis assuruerunt dicti Raginaldus et Petrus, nominibus quibus supra, et confessi sunt. De quibus sex viginti sex libris et septem solidis receptis, ut predicitur, quitaverunt dicti Radulphus et Petrus, nominibus quibus supra, dictos dominum ducem et dominum Petrum et dictos executores, hoc salvo quod recunsum ${ }^{140}$ habere possint de eo quod dicebant et debere satisfieri de dicta pecunie summa, in bona moneta currente, inspecto tempore captionis predicte. Datum teste sigillo curie Redonensis una cum sigillo dicti Raginaldi, pro se et dicto Petro, nominibus quibus supra, die lune post Indica me, anno Domini $\mathrm{M}^{\circ} \mathrm{CCC}^{\circ}$ sexto.

Sachent tous que devant nous, l'official de Rennes, en droit personnellement comparus, Renaud Gicquel, citoyen de Rennes, tant en son nom qu'au nom de Pétronille, autrefois femme de Jean Leclavereul et maintenant du dit Renaud, et aussi Pierre, dit Clavereul, fils des dits Jean et Pétronille, tant en son nom qu'en celui de Jean et Jean, enfants des dits Jean et Pétronille, ont confessé avoir eu et reçu des exécuteurs du seigneur défunt d'illustre souvenir Jean, récemment duc de Bretagne et comte de Richemont, 126 livres et 7 sous de faible monnaie, pour ce que le noble homme, le seigneur Pierre de Bretagne, chevalier, fils du dit seigneur duc, avait fait prendre et avait eu des sommes des dits Jean et Renaud, contre la volonté des mêmes, qu'ils avaient dénoncé avec plainte au dit seigneur duc, du temps où il vivait, et somme d'argent de laquelle il y avait mention dans un rouleau remis aux mêmes exécuteurs par la partie du dit seigneur Pierre, et que la prise avait été faite il y a douze ans écoulés ou environ, preuve suffisante ayant été faite là-dessus devant les susdits exécuteurs, ainsi que nous l'ont assuré les dits Renaud et Pierre, au nom des dits ci-dessus, et ils l'ont confessé. 126 livres et 7 sous reçus, comme il est dit, desquels les dits Raoul et Pierre ont tenu quitte, au nom des dits ci-dessus, les dits seigneur duc, seigneur Pierre et les

137. Mention dorsale d'une main du XIX s. : « 13 mars 1307. Quittance de 126 livres payées à Renaud, Gui et Jean Le Clavereul. "

138. Il y a cependant une erreur d'attribution d'acte, donné par Marie GAONACH comme étant une quittance de 23 sous payés à la veuve de Michel Leroy.

139. "summa ", écrit au-dessus de la ligne, $A$.

140. Sic, $A$, pour "retunsum». 
dits exécuteurs, sauf ceci : qu'ils puissent avoir le rabat de ce qu'ils disaient et devoir être satisfaits de la dite somme d'argent en bonne monnaie courante, vu le temps de la susdite prise. Donné témoin le sceau de la cour de Rennes, en même temps que le sceau du dit Renaud, pour lui et le dit Pierre, au nom des dits ci-dessus, le lundi après Indica me, en l'an du Seigneur mille deux cent trois cent six.

$-6-$

1307 (n. st.), 20 mars.

L'official de la cour de Rennes fait savoir que Jean Dupuis a reçu des exécuteurs testamentaires du duc Jean 12 livres de faible monnaie, en compensation du vin que lui avaient pris les gens de Pierre de Bretagne.

A. Original, parchemin, larg. $195 \mathrm{~mm} \times$ haut. $50 / 35 \mathrm{~mm}$ (haut. de queue, $15 \mathrm{~mm}$ ), scellé sur simple queue de parchemin du sceau de l'official de Rennes en cire verte $^{141}$, Arch. dép. de Loire-Atlantique, E 22, nº 18.

INDIQUE : GAONACH, Marie, Jean II..., op. cit., p. 205.

Universis presentes litteras inspecturis vel audituris, officialis curie Redonensis, salutem in Domino. Noveritis quod coram nobis in jure personaliter constitutus, Johannes dou Puiz, aliter dictus Cartisinne, confessus fuit coram nobis se habuisse et recepisse ab executoribus inclite recordacionis domini Johannis, quondam ducis Britannie et comitis Richemundie, per manum Rolandi Lumbardi, duodecim libras debilis monete, pro recompensacione facione vini dicti Johannis, capti per gentes domini Petri de Britannia, de quibus idem Johannes habebat litteras domini Petri predicti. De quibus duodecim libris dictus Johannes se a dictis domino duce et ejus executoribus tenuit penitus pro pagato et de quibus quitavit penitus et expresse dictos dominum ducem et ejus executores. Datum die lune ante Ramos palmarum, anno Domini $\mathrm{M}^{\circ} \mathrm{CCC}^{\circ}$ sexto.

À tous ceux qui verront ou entendront les présentes lettres, l'official de la cour de Rennes, salut dans le Seigneur. Sachez que, devant nous personnellement comparu en droit, Jean Dupuis, autrement dit Cartisinne, a confessé devant nous avoir eu et reçu des exécuteurs du seigneur Jean d'illustre souvenir, feu duc de Bretagne et comte de Richemont, par la main de Roland le Lombard, 12 livres de faible monnaie, pour compensation faite du vin du dit Jean, pris par les gens du seigneur Pierre de Bretagne, dont le même Jean avait des lettres du susdit seigneur Pierre. Desquelles 12 livres le dit Jean s'est pleinement tenu pour payé des dits seigneur duc et de ses exécuteurs et desquelles il a tenu quitte pleinement et expressément les dits seigneur duc et ses exécuteurs. Donné le lundi avant les Rameaux, en l'an du Seigneur mille trois cent six.

1307, 31 mars.

L'official de Vannes fait savoir qu'Eudes Frelon, qui avait porté plainte en son nom et celui de son père pour les ravages causés à ses bois et vignes par Pierre de Bretagne et ses gens, a transigé avec les exécuteurs testamentaires de Jean II pour un dédommagement de 100 livres de bonne monnaie.

141. Il ne reste qu'un fragment de ce sceau. Par ailleurs, mention dorsale du XIXe siècle : «1307. Reçu de 12 livres payées à Jean Dupuis pour vin pris ». 
A. Original, parchemin, scellé sur deux simples queues de parchemin du sceau de l'official de Vannes et du sceau d'Eudes Frelon, tous deux en cire verte ${ }^{142}$, Arch. dép. de Loire-Atlantique, E 22, nº 20.

INDIQUE : GAONACH, Marie, Jean II..., op. cit., p. 205.

Universis presentes licteras inspecturis et audituris, officialis curie Venetensis, salutem in Domino. Noverint universi quod coram nobis personaliter constitutus, Eudo Frellon, de dyocese redonensi, se scienter subiciens juridictioni nostre, quoad ea que sequitur confessus fuit, quod super sectione, deportacione, et devastacione et capcione quorumdam nemorum et vinorum, tam patris sui quondam, quam suorum alias, factis ut asserebat, per nobilem virum dominum Petrum de Britannia et gentes suas, injuste et contra voluntatem dictorum patris et Eudonis super quibus fuerat requisitus de faciendo fieri sibi eisdem justicie complementum dominus Johannes, quondam dux Britannie, et super hoc negligens et remissus; et super hiis et dampnis, et deperditis et expensis, in prosequtione premissorum deposuerat dictus Eudo, tam nomine suo quam nomine patris predicti, cujus est heres querimoniae erga exequtores dicti ducis finaverat et amicabiliter composuerat cum exequtoribus predictis ad centum libras bone monete quas confessus fuit se habuisse et recepisse a dictis exequtoribus per manum Rollandi Le Lambart; et de premissis se tenuit pro pagato et ${ }^{143}$ quitavit super premissis dictos ducem, et heredes et executores suos, et dictum dominum Petrum et suos. Datum teste sigillo curie nostre, una com $^{144}$ sigillo dicti Eudonis, die veneris post Rexurrectionem Domini, anno ejusdem $\mathrm{M}^{\circ}$ $\mathrm{CCC}^{\mathrm{mo}}$ septimo.

À tous ceux qui verront et entendront les présentes lettres, l'official de la cour de Vannes, salut dans le Seigneur. Sachent tous que, ayant devant nous personnellement comparu, Eudes Frelon, du diocèse de Rennes, se soumettant sciemment à notre juridiction, dans la mesure où il a confessé ce qui suit, que, au sujet de la coupe, du transport, de la dévastation et de la prise de bois et de vins, tant de feu son père qu'ailleurs des siens, commis, ainsi qu'il l'assurait, par le noble homme, le seigneur Pierre de Bretagne et ses gens, injustement et contre la volonté des dits père et Eudes, au sujet de quoi le seigneur Jean, feu duc de Bretagne, avait été requis de faire faie exécution de justice pour les mêmes, et fut négligent et indifférent à ce sujet; et sur ceci, les dommages, pertes et dépenses, le dit Eudes avait déposé pour la poursuite des susnommés, tant en son nom qu'au nom de son susdit père, dont il est héritier de la plainte, auprès des exécuteurs du dit duc, il s'est arrangé et a amicalement composé avec les exécuteurs susdits pour 100 livres de bonne monnaie qu'il a confessé avoir eues et reçues des dits exécuteurs par la main de Roland Le Lombard; et il se tint pour payé de ce qui est susdit et en a tenu quitte les dits duc, ses héritiers et exécuteurs, et le dit seigneur Pierre et les siens. Donné témoin le sceau de notre cour, en même temps que le sceau du dit Eudes, le vendredi après la Résurrection du Seigneur, en l'an du même mille trois cent sept.

142. Il ne reste qu'un fragment de ce sceau. Par ailleurs, mention dorsale du XIV ${ }^{\mathrm{e}}$ siècle : "Et est sciendum quod si alia littera erat absoluta vel concessa seu [...] de tanta summa super isto facto vel consimili, quae est annullata " (Et il est à savoir que si une autre lettre était délivrée ou concédée ou [...] d'une si grande somme sur ce fait ou un similaire, qu'elle soit nulle). Il y a également une mention dorsale du XIXe siècle : " 1307 . Reçu de 100 livres payées à Eudes Frelon pour dégâts sur ses terres ".

143. " et ", répété une fois, $A$.

144. Sic, $A$, pour " cum». 
1308 (n. st.), 23 mars.

Geoffroy Choan, clerc, a eu des exécuteurs testamentaires de Jean II 60 livres de bonne monnaie courante pour ce que le devait Pierre de Bretagne.

A. Original, parchemin, larg. 165/160 $\mathrm{mm}$ x haut. 80/75 mm (haut. de queue, $5 \mathrm{~mm}$ ), scellé sur simple queue de parchemin du sceau de Geoffroy Choan en cire verte ${ }^{145}$, Arch. dép. de Loire-Atlantique, E $23, n^{\circ} 28$.

INDIQUE : GAONACH, Marie, Jean II..., op. cit., p. 209.

Noverint universi quod ego, Gauffridus Choan, clericus, habui et recepi ab exequtoribus inclite recordationis, bone memorie Johannis, quondam ducis Britannie ${ }^{146}$, comitisque Richemondie, ultimo deffuncti, per manum Rollandi Lanbardi, sexaginta libras bone monete usualis, pro domino Petro de Britannia, filio dicti ducis et de mandato ipsius domini Petri, per litteras suas, prout in ipsis litteris quibus presens littera est annexa continetur, velut sexaginta una libras alias mihi ab predictis exequtoribus solutas, de quibus habent licteras quitacionis sigillo meo sigillatas, quitans dictum dominum ducem et ejus exequtores ac dictum dominum Petrum de dictis sexaginta libris. Datum teste sigillo meo proprio presentis licteris apposito, die sabbati ante Annunciacionem Beate Marie Virginis, anno Domini millesimo trecentesimo septimo.

Sachez tous que moi, Geoffroy Choan, clerc, ai eu et reçu des exécuteurs de Jean, d'illustre souvenir, de bonne mémoire, feu duc de Bretagne et comte de Richemont, récemment défunt, par la main de Roland Le Lombard, 60 livres de bonne monnaie usuelle, pour le seigneur Pierre de Bretagne, fils du dit duc, et sur ordre du seigneur Pierre lui-même, par ses lettres, ainsi que cela est contenu dans les lettres mêmes auxquelles la présente lettre est annexée, comme 61 autres livres à moi versées par les susdits exécuteurs, dont ils ont des lettres de quittance scellées de mon sceau, quittant le dit seigneur duc, ses exécuteurs et le dit seigneur Pierre des dites 60 livres. Donné témoin mon sceau propre apposé aux présentes lettres, le samedi avant l'Annonciation de la sainte Vierge Marie, en l'an du Seigneur mille trois cent sept.

$$
\mathbf{- 9}-
$$

1308 (n. st.), 3 mai.

Guillaume Le Sergent a reçu des exécuteurs testamentaires ducaux 11 livres de bonne monnaie en compensation d'un cheval que lui avait pris Pierre de Bretagne, et pour lequel il avait déjà réclamé auprès du défunt duc Jean II.

145. Mention d'une main du XIX siècle : « Reçu de 60 livres payées à Geoffroy Chouan, clerc ». Cet acte est lié à un autre acte en parchemin, un mandement de Pierre de Bretagne aux exécuteurs de payer à Geoffroy Choan 60 livres de bonne monnaie courante (Arch. dép. de Loire-Atlantique, E 23, n² 29), mandement publié par GAONACH, Marie, Jean II..., op. cit., p. 186.

146. Suivi de "ultimo deffuncti », barré, $A$. 
A. Original, parchemin, larg. 190/180 mm x haut. 115/90 mm (haut. de queue, $25 \mathrm{~mm}$ ), scellé sur simple queue de parchemin du sceau de Guillaume Le Sergent en cire verte ${ }^{147}$, Arch. dép. de Loire-Atlantique, E $21, \mathrm{n}^{\circ} 74$.

IndiQUE : GAONACH, Marie, Jean II..., op. cit., p. 208.

Noverint universi quod ego Guillelmus, dictus serviens, de Guengamp, habui et recepi ab exequtoribus testamenti inclite recordacionis Johannis, quondam ducis Britannie, comitisque Richemundie, ultimo deffuncti, per manum Rollandi Lumbardi de Kemperelé, undecim libras bone monete currentis, pro quodam equo, quem nobilis vir, dominus Petrus de Britannia, filius ejusdem ducis, habuerat per violenciam michi ab eodem factam et contra meam propriam voluntatem; et quem equm ${ }^{148}$ ego prosequtus fui usque ad domum dicti domini ducis de Benestio, in insula de Ruysio, quem equm ibidem inveni cum quodam serviente dicti domini ducis, $[\ldots]^{149}$; cum instantia a dicto duce dictum equm mihi restitui, a me per violentiam sit ablatum; qui quidem dominus dux istud mihi renuit facere, dicens et respondens mihi quod dictum equm non haberem; que omnia ista dictis exequtoribus per bones testes probavi, et de dictis undecim libris me teneo pro pagato, et quito et absolvo prefatos, dictum ducem, et ejus exequtores et heredes de dictis denariis et equo et specialiter dictum dominum Petrum. Datum teste sigillo meo proprio, presentis litteris apposito, die martis ante cathedra sancti Petri, anno Domini $\mathrm{M}^{\circ} \mathrm{CCC}^{\mathrm{mo}}$ septimo.

Sachent tous que moi, Guillaume, dit Le Sergent, de Guingamp, j'ai eu et reçu des exécuteurs du testament de Jean d'illustre souvenir, feu duc de Bretagne et comte de Richemont, récemment défunt, par la main de Roland Le Lombard, de Quimperlé, 11 livres de bonne monnaie courante, pour un cheval que le noble homme, le seigneur Pierre de Bretagne, fils du même duc, avait eu par violence à moi faite et contre ma propre volonté; cheval que j'ai suivi jusqu'à la demeure du dit seigneur duc de Benester, dans l'île de Rhuys, cheval que j'y ai trouvé avec un serviteur du dit seigneur duc, en usant sur le chemin comme d'un animal; demandant instamment au dit duc que le dit cheval à moi retiré par violence me fut restitué, le seigneur duc refusa de le faire, disant et me répondant que je n'aurai pas le dit cheval; tout ceci, je l'ai prouvé aux dits exécuteurs par bons témoins, et je me tiens pour payé des dites 11 livres, et je tiens quitte et délie les susdits, le dit duc et ses dits exécuteurs et héritiers des dits deniers et cheval, et spécialement le dit seigneur Pierre. Donné témoin mon propre sceau, apposé aux présentes lettres, le mardi avant la Chaire de saint Pierre, l'an du Seigneur mille trois cent sept.

$$
-10-
$$

1308 (n. st.), 3 mai.

L'official de Rennes fait savoir que Guillaume, recteur de Lécousse, et Jeanne, sa mère, victimes de pillages dus à Pierre de Bretagne et ses gens, notamment dans leur maison de Saint-Aubin-du-Cormier, se sont finalement arrangés avec les exécuteurs testamentaires de Jean II pour un dédommagement de 30 livres de monnaie courante;

147. À la base de la simple queue, il est inscrit : "Solutio per manum Rollandi Lombardi " (versement par la main de Rolland Le Lombard). Mention dorsale d'une main du XIXe siècle : " 1308. Reçu de 11 livres payées pour l'enlèvement d'un cheval à Guillaume, dit Le Sergent, de Guingamp ".

148. " equm ", sic, $A$, pour " equum ". Cette graphie est d'emploi constant dans ce texte. 149. Ici manque une petite incise que ni moi, ni les nombreuses personnes à qui j'ai soumis le texte, n'avons correctement réussi à transcrire. 
en échange de quoi, ils abandonnent toutes poursuites contre les exécuteurs et le duc Arthur II, mais pas contre Pierre de Bretagne, ses serviteurs et toute autre personne responsable.

A. Original, larg. 230/240 $\mathrm{mm} \times$ haut. $150 / 120 \mathrm{~mm}$ (haut. de queues, respectivement $20 \mathrm{~mm}$ et $10 \mathrm{~mm}$ ), scellé sur deux simples queues de parchemin du sceau de la cour officiale de Rennes et de celui de Guillaume, recteur de Lécousse, tous deux en cire verte ${ }^{150}$, Arch. dép. de Loire-Atlantique, E23, ${ }^{\circ} 1$.

INDIQUE : GAONACH, Marie, Jean II..., op. cit., p. 208.

Coram nobis, officialis Redonensis, personaliter constituti, Guillelmus, rector ecclesie de Exusa et Johanna, ejus mater, vidua, confessi sunt super eo quod ipsi apud executores inclite recordacionis domini Johannis, quondam dux ${ }^{151}$ Britannie, novisime ${ }^{152}$ defuncti, detulerant querimoniam pro eo quod cum dudum dominus Petrus de Britannia, prefati ducis filius, averia, fena, avenam et nonnulla alia bona dictorum Guillelmi et ejus matris capi fecisset et a quadam domo dicti Guillelmi apud Sanctum Albinum de Cormerio sita per dicti Petri servientes fracta quemdam equm, lanam, coverturas, linteamena, couechera, auricularia et alia bona ipsius Guillelmi exheri fecerat et apud manerium de Mota deportari et per plurimas violencias eisdem matri et filio intulerat et inferri fecerat injuste dictusque Guillelmus prefatum ducem super eo adierat et eidem justiciam de dicto filio suo petierat super premissis, quam eidem negavit aut saluti minus juste procraxit ${ }^{153}$ in hac parte et manifeste defecerat dux prefatus. Tandem inter prefatos executores, ex una parte, et dictam viduam et ejus filium, ex altera compositum extitit in hunc modum, quod si dux ipse, in hac parte negligentiam aliquam culpabilem commiserat, aut ea occasione eisdem matri et filio jure poli vel fori in aliquo tenebatur, dicti mater et ejus filius predictus, pro triginta libras monete currentis indulgeant et renunciant eidem domino duci cujusmodi indulgenciam sic fecisse et pecuniam ab eisdem executoribus recepisse predictam confessi sunt vidua et ejus filius predicti, cedentes eciam executoribus antedictis et domino duci moderno omnes actiones et jura sibi competentia pro dampnis et bonis occupatis predictis, usque ad dictam peccunie summam, salva eisdem matre et ejus filio super residuo premissorum et singulorum prosecucione juris sui contra dictum Petrum ejusque servientes et alios quosque qui occasione premissorum sunt eisdem in aliquo obligati, cujusmodi compositionem inviolabiliter observare promiserant vidua et ejus filius predicti, et ad premissa tenenda per nos finaliter extiterunt condempnari. Datum die veneris post festum Philippi et Jacobi apostolorum, anno Domini $\mathrm{M}^{\circ} \mathrm{CCC}^{\mathrm{mo}}$ octavo. Datum teste sigillo nostre curie, una cum sigillo dicti Guillelmi.

Devant nous, official de Rennes, personnellement comparus, Guillaume, recteur de l'église de Lécousse, et Jeanne, sa mère, veuve, ont confessé sur le fait qu'euxmêmes avaient déposé plainte auprès des exécuteurs du seigneur d'illustre souvenir Jean, feu duc de Bretagne, récemment défunt, pour ce que naguères le seigneur Pierre de Bretagne, fils du susdit duc, avait fait prendre du bétail, du foin, de l'avoine et

150. Il ne reste que des fragments de ces sceaux. L'original, dans son ensemble, présente quant à lui une tache noircie en son extrémité supérieure. Mention d'une main du XIX siècle : « 1308. Reçu de 30 livres payés à Guillaume, recteur de Lécousse et à sa mère pour indemnité de mobilier enlevé. "

151. "dux " sic A, pour "ducis ".

152. Sic, A, pour "novissime"

153. Sic, $A$, pour " protraxit ". 
quelques autres biens des dits Guillaume et sa mère, avait fait retirer d'une maison du dit Guillaume sise à Saint-Aubin-du-Cormier, fracturée par des serviteurs du dit Pierre, un cheval, de la laine, des couvertures, des toiles de lin, de la literie, un lit et d'autres biens de Guillaume lui-même, les avait fait transporter au manoir de La Motte et avait fait infliger et porter injustement plusieurs violences aux mêmes mère et fils; et le dit Guillaume était allé à ce sujet auprès du susdit duc et lui avait demandé justice de son dit fils sur ce qui est susdit, ce qu'il refusa au même ou par le salut protégea moins justement dans cette affaire, et le susdit duc avait manifestement commis une faute. Enfin, entre les susdits exécuteurs, d'une part, et ladite veuve et son fils, de l'autre, il se fit une composition en cette manière, que si le duc même avait commis en cette affaire quelque négligence coupable, ou était tenu en cette occasion envers les mêmes mère et fils, par droit coutumier ou naturel, en quoi que ce fut, les dits mère et son fils susdit n'exigeaient pas et renonçaient pour 30 livres de monnaie courante, et la veuve et son fils susdits ont confessé n'avoir fait d'exigence de cette sorte envers le même seigneur duc et avoir reçu le susdit argent des mêmes exécuteurs testamentaires, retirant aussi vis-à-vis des susdits exécuteurs et de l'actuel duc de Bretagne toutes actions et droits appropriés pour les susdits dommages et biens occupés, jusqu'à la dite somme d'argent, sauf la poursuite de leur droit, par les mêmes mère et son fils, sur le reste de ce qui est susdit et chaque point particulier, contre le dit Pierre, ses serviteurs et les autres qui, à l'occasion de ce qui est susdit, sont en quoi que ce soit responsables vis-à-vis d'eux; composition de cette sorte que la veuve et son fils susdits avaient promis d'observer inviolablement, et ils ont été finalement condamnés par nous à tenir ce qui est susdit. Donné le vendredi après la fête des apôtres Philippe et Jacques, en l'an du Seigneur mille trois cent huit. Donné, témoin le sceau de notre cour, en même temps que le sceau du dit Guillaume. 


\section{RÉSUMÉ}

Pierre de Bretagne, fils puîné du duc Jean II de Bretagne (1286-1305) a été longtemps laissé de côté, peu mis en valeur par les historiens successifs de la Bretagne médiévale, mis à part le fameux épisode de son emprisonnement pour dettes à Paris en 1293. L'examen de l'ensemble des sources subsistant à son sujet permet de découvrir un personnage inhabituellement détonnant, comparé à la famille ducale de Dreux don il est issu : cadet visiblement dépensier et sans scrupule, il accumule les dettes, ravages et déprédations, tant dans le duché de Bretagne que sur la place de Paris. Amateur de vins et de chevaux, il est tout à la fois débiteur auprès de marchands pour plusieurs centaines de livres qu'auprès de modestes veuves pour quelques dizaines de sous, et bénéficie en outre, malgré les plaintes, de la protection ducale.

La disparition inattendue de son père en 1305 lui a offert l'opportunité inespérée de liquider le colossal passif qu'il avait accumulé, les exécuteurs testamentaires ducaux ayant notamment pour mission de régler toutes ses dettes. C'est à cette longue suite de paiements que nous devons la connaissance dudit " Pierres, jadis fiulz dou duc de Bretaigne et conte de Richemond ", prodigue et pillard au gré de ses envies.

\section{ABSTRACT}

Peter of Brittany, the youngest son of John II, duke of Brittany (1286-1305), has - with the exception of his famous imprisonment for debt in Paris, in 1293, been widely by successive historians of Brittany. The review of all existing sources about him unexpectedly reveals a person surprisingly at odds with the ducal family he was born into. He was a spendthrift, accumulating debts, engaging in acts of vandalism and depredations, whether in France or in Paris. As a lover of wine and horses, he was indebted to the tune of several hundreds of pounds to merchants, but also owed small sums to humble widows. Nevertheless, he was able to call upon the protection of the duke, despite their complaints.

His father's unexpected death, in 1305, was an unforeseen opportunity to pay off all these outstanding amounts, as the ducal executors were required to repay his debts. Thanks to that long list of payments, we can understand more fully "Pierres, jadis fiulz dou duc de Bretaigne et conte de Richemond", the prodigal and erstwhile looter. 